\title{
A solar storm observed from the Sun to Venus using the STEREO, Venus Express, and MESSENGER spacecraft
}

Article

Published Version

Rouillard, A. P., Davies, J. A., Forsyth, R. J., Savani, N. P., Sheeley, N. R., Thernisien, A., Zhang, T.-L., Howard, R. A., Anderson, B., Carr, C. M., Tsang, S., Lockwood, M., Scott, C. J., Harrison, R. A., Bewsher, D., Fränz, M., Crothers, S. R., Eyles, C. J., Brown, D. S., Whittaker, I., Hapgood, M., Coates, A. J., Jones, G. H., Grande, M., Frahm, R. A. and Winningham, J. D. (2009) A solar storm observed from the Sun to Venus using the STEREO, Venus Express, and MESSENGER spacecraft. Journal of Geophysical Research, 114 (A7). A07106. ISSN 0148-0227 doi: https://doi.org/10.1029/2008JA014034 Available at https://centaur.reading.ac.uk/7216/

It is advisable to refer to the publisher's version if you intend to cite from the work. See Guidance on citing.

Published version at: http://dx.doi.org/10.1029/2008JA014034

To link to this article DOI: http://dx.doi.org/10.1029/2008JA014034

Publisher: American Geophysical Union

Publisher statement: "An edited version of this paper was published by AGU.

Copyright (2009) American Geophysical Union." 
All outputs in CentAUR are protected by Intellectual Property Rights law, including copyright law. Copyright and IPR is retained by the creators or other copyright holders. Terms and conditions for use of this material are defined in the End User Agreement.

\section{www.reading.ac.uk/centaur}

\section{CentAUR}

Central Archive at the University of Reading

Reading's research outputs online 


\title{
A solar storm observed from the Sun to Venus using the STEREO, Venus Express, and MESSENGER spacecraft
}

\author{
A. P. Rouillard, ${ }^{1,2}$ J. A. Davies, ${ }^{2}$ R. J. Forsyth,${ }^{3}$ N. P. Savani, ${ }^{3}$ N. R. Sheeley, ${ }^{4}$ \\ A. Thernisien, ${ }^{4}$ T.-L. Zhang, ${ }^{5}$ R. A. Howard ${ }^{4}$ B. Anderson, ${ }^{6}$ C. M. Carr, ${ }^{3}$ S. Tsang, ${ }^{7}$ \\ M. Lockwood, ${ }^{1,2}$ C. J. Davis, ${ }^{2}$ R. A. Harrison, ${ }^{2}$ D. Bewsher, ${ }^{2,8}$ M. Fränz, ${ }^{9}$ S. R. Crothers, ${ }^{2}$ \\ C. J. Eyles ${ }^{2,10}$ D. S. Brown, ${ }^{8}$ I. Whittaker, ${ }^{8}$ M. Hapgood, ${ }^{2}$ A. J. Coates, ${ }^{7}$ G. H. Jones, ${ }^{7}$ \\ M. Grande, ${ }^{8}$ R. A. Frahm, ${ }^{11}$ and J. D. Winningham ${ }^{11}$
}

Received 6 January 2009; revised 7 April 2009; accepted 15 April 2009; published 21 July 2009.

[1] The suite of SECCHI optical imaging instruments on the STEREO-A spacecraft is used to track a solar storm, consisting of several coronal mass ejections (CMEs) and other coronal loops, as it propagates from the Sun into the heliosphere during May 2007. The 3-D propagation path of the largest interplanetary CME (ICME) is determined from the observations made by the SECCHI Heliospheric Imager (HI) on STEREO-A (HI-1/2A). Two parts of the CME are tracked through the SECCHI images, a bright loop and a V-shaped feature located at the rear of the event. We show that these two structures could be the result of line-of-sight integration of the light scattered by electrons located on a single flux rope. In addition to being imaged by HI, the CME is observed simultaneously by the plasma and magnetic field experiments on the Venus Express and MESSENGER spacecraft. The imaged loop and V-shaped structure bound, as expected, the flux rope observed in situ. The SECCHI images reveal that the leading loop-like structure propagated faster than the V-shaped structure, and a decrease in in situ CME speed occurred during the passage of the flux rope. We interpret this as the result of the continuous radial expansion of the flux rope as it progressed outward through the interplanetary medium. An expansion speed in the radial direction of $\sim 30 \mathrm{~km} \mathrm{~s}^{-1}$ is obtained directly from the SECCHI-HI images and is in agreement with the difference in speed of the two structures observed in situ. This paper shows that the flux rope location can be determined from white light images, which could have important space weather applications.

Citation: Rouillard, A. P., et al. (2009), A solar storm observed from the Sun to Venus using the STEREO, Venus Express, and MESSENGER spacecraft, J. Geophys. Res., 114, A07106, doi:10.1029/2008JA014034.

\section{Introduction}

[2] Coronal mass ejections (CMEs), large-scale eruptions of solar material expelled into the solar corona, are one of the most spectacular solar phenomena [Hudson et al., 2006]. CMEs expand outward from the Sun through the solar wind plasma of the interplanetary medium. Solar ejecta, such as CMEs or streamer disconnection events, involve the release of plasma and complex helical magnetic

\footnotetext{
${ }^{1}$ Space Environment Physics Group, School of Physics and Astronomy, University of Southampton, Southampton, UK.

${ }^{2}$ Space Science and Technology Department, Rutherford Appleton Laboratory, Chilton, UK.

${ }^{3}$ Space and Atmospheric Physics, Blackett Laboratory, Imperial College London, London, UK USA.

${ }^{4}$ Space Science Division, Naval Research Laboratory, Washington, D. C.,

${ }^{5}$ Space Research Institute, Austrian Academy of Sciences, Graz, Austria.

Copyright 2009 by the American Geophysical Union.

0148-0227/09/2008JA014034\$09.00
}

fields that are often associated with changes in the location of the streamers near to the Sun [Suess et al., 2004], and that generally disrupt the more regular spiral distribution of alternating fast and slow solar wind in the interplanetary medium [Burlaga, 1990; Burlaga et al., 1991]. CMEs can provide a significant fraction of the average mass flux to the near-ecliptic solar wind, nearly $15 \%$ at solar maximum [Webb and Howard, 1994].

[3] The rarity of spacecraft in the region between the Sun and the Earth, together with the limited extent of corona-

${ }^{6}$ Johns Hopkins University Applied Physics Laboratory, Baltimore, Maryland, USA.

${ }^{7}$ Mullard Space Science Laboratory, University College London, Dorking, UK.

${ }^{8}$ Institute of Mathematics and Physics, Aberystwyth University, Aberystwyth, UK.

${ }^{9}$ Max-Planck-Insitut für Sonnensystemforschung, Katlenburg-Lindau, Germany.

${ }^{10}$ Grupo de Astronomia y Ciencias del Espacio, Universidad de Valencia, Valencia, Spain.

${ }^{11}$ Southwest Research Institute, San Antonio, Texas, USA. 
graphs' fields of view, mean that prior to the launch of the STEREO mission very few studies had considered the coupling between the background solar wind and solar transients between the Sun and 1 AU. Yet a detailed assessment of the role and importance of solar wind transients in affecting the properties of the solar wind near 1 AU has important consequences for our understanding of short- and long-term changes in solar wind-magnetosphere coupling [Borovsky and Denton, 2006; Lavraud et al., 2006; Lavraud and Borovsky, 2008; Pulkkinen et al., 2007] and the modulation of galactic cosmic rays [Burlaga et al., 2003]. Past studies have shown, for instance, that large CMEs that interact significantly with the slow background solar wind are more geoeffective than CMEs that propagate in a uniform fast solar wind [Burlaga et al., 1987].

[4] The consequential dichotomy of solar wind transient studies carried out using either in situ measurements or by using coronagraphs has led to the largest transients being termed coronal mass ejections near the Sun and interplanetary coronal mass ejections (ICMEs) by the time they are measured in situ.

[5] The coronagraphs onboard the Solar and Heliospheric Observatory (SoHO) [Brueckner et al., 1995] have provided nearly continuous observations of solar ejecta for more than a decade. These coronagraph observations have allowed detailed investigations of the kinematics, dynamics and energetics of CMEs near the Sun [e.g., Sheeley et al., 1999; Vourlidas et al., 2000; Gopalswamy et al., 2001; Suess et al., 2004; Cremades and Bothmer, 2004; Thernisien et al., 2006].

[6] ICMEs are frequently observed in situ, by plasma analyzers and magnetometers. A subset of solar wind magnetic flux ropes stand out in the in situ measurements; these were termed magnetic clouds (MCs) by Burlaga et al. [1981]. MCs are characterized by an enhanced magnetic field strength, smooth rotation of the magnetic field vector, and low proton density and temperature, and are the possible manifestation of a CME. The association between CMEs and MCs was made when a MC was observed by Helios 1 a few days after being observed as a CME by the SOLWIND coronagraphs onboard the U.S. Air Force P78-1 satellite [Burlaga et al., 1982; Sheeley et al., 1985]. The typical structure observed for a fast CME by a satellite such as ACE is a fast-mode shock wave followed by a dense (and hot) sheath of plasma (the downstream region of the shock) and a magnetic cloud [Burlaga et al., 1981].

[7] Here, we present the results of a case study in which a CME is continuously tracked from the Sun out to Venus, using the imaging instrumentation onboard the STEREO spacecraft. Large-scale loops, small coronal loops, V-shaped structures and plasma blobs are various coronal structures observed in the white light images during this solar event. The largest structure is tracked to the Venus Express (VEX) and MESSENGER spacecraft, providing an excellent opportunity to compare the CME and in particular its white light appearance with in situ observations.

\section{Instruments}

[8] The two spacecraft of the NASA STEREO mission, STEREO-A and STEREO-B, carry near-identical instru- mentation that includes the SECCHI imaging package. SECCHI [Howard et al., 2008] comprises an extreme ultraviolet imager (EUVI), two coronagraphs (COR-1 and COR-2), and the heliospheric imagers (HI). The HI instrument [Eyles et al., 2009] on each STEREO spacecraft consists of two white light, wide-field imagers, HI-1 and HI-2 which, like coronagraphs, detect sunlight scattered from coronal electrons by Thomson scattering [Vourlidas and Howard, 2006]. The inner HI-1 cameras have a $20 \times$ $20^{\circ}$ field of view (fov) that extends from 4 to $24^{\circ}$ of elongation in the ecliptic plane. The $70^{\circ}$ fov of the outer HI-2 cameras extends from 18.7 to $88.7^{\circ}$ of elongation along the ecliptic. Figure 1a presents a view of the ecliptic plane from above on 21 May 2007 showing the relative positions of STEREO-A (A) and the Earth (E). The elongation extent in the ecliptic plane of the fovs of COR-2, HI-1 and HI-2 on STEREO-A, termed COR-2A, HI-1A and HI-2A, are marked by green, red and blue lines, respectively. Figure $1 \mathrm{~b}$ shows the full extent of the fovs of COR-2A (green circle), HI-1A (red square) and HI-2A (blue circle) in elongation and elevation, $\varepsilon$ (the angle out of the ecliptic plane). In addition, the positions of Venus $(\mathrm{V})$ and MESSENGER (M) are indicated; the intersection of the solar equatorial plane and the solar meridional plane passing through Venus is also plotted as a solid black line.

\section{SECCHI Observations of the CME Propagation}

\subsection{Formation of the Solar Storm in COR-2A}

[9] A CME is observed to takeoff from position angle (PA) $\sim 90^{\circ}$ on 21 May 2007 in COR-1A/B images (not shown). PA is the angle in degrees from solar north $\left(=0^{\circ}\right)$ such that east $=90^{\circ} \mathrm{PA}$, south $=180^{\circ} \mathrm{PA}$ and west $=270^{\circ} \mathrm{PA}$. A large loop-like structure emerges at 0750 UT followed by an almost circular structure which develops into a $\mathrm{V}$ shape as it propagates outward. These two structures move out of the field of view of COR-1A/B at around 2000 UT. As the $\mathrm{V}$-shaped structure propagates outward, a second smaller parcel of dense plasma emerges at a larger PA $\left(\sim 94^{\circ}\right)$ and appears to propagate along the southern flank of the first event. A streamer is observed "face-on" on the eastern limb of the Sun during the takeoff of this main event indicating that the streamer belt is undergoing a latitudinal excursion.

[10] The CME enters the COR-2A/B fov at 1000 UT on 21 May. The sequence of COR-2A running difference images shown in Figure 2 reveals the state of the corona several hours before the main CME passage (Figure 2a), during CME takeoff (Figures 2b, 2c, and 2d), and after the main CME passage (Figures $2 \mathrm{e}$ and $2 \mathrm{f}$ ). The leading loop-like structure and following $\mathrm{V}$-shaped structure of the CME observed by COR-1A/B can also be identified in the COR-2A/B images. The former is labeled $\mathrm{A}$ in Figures $2 \mathrm{~b}$ and $2 c$, and the latter is labeled B (Figures $2 b, 2 c$, and $2 d$ ); these features are, as will be shown later, well resolved by HI. We associate structures A and B with different parts of the same transient. Other transients that also alter significantly the coronal brightness are identified. Features $\mathrm{C}$ and $\mathrm{D}$ (Figures $2 \mathrm{c}$ and $2 \mathrm{~d}$ ) are part of the smaller event, also seen by COR-1A/B. The system comprising features $\mathrm{C}$ and $\mathrm{D}$ appears to interact with the V-shaped structure B in Figure 2c, 


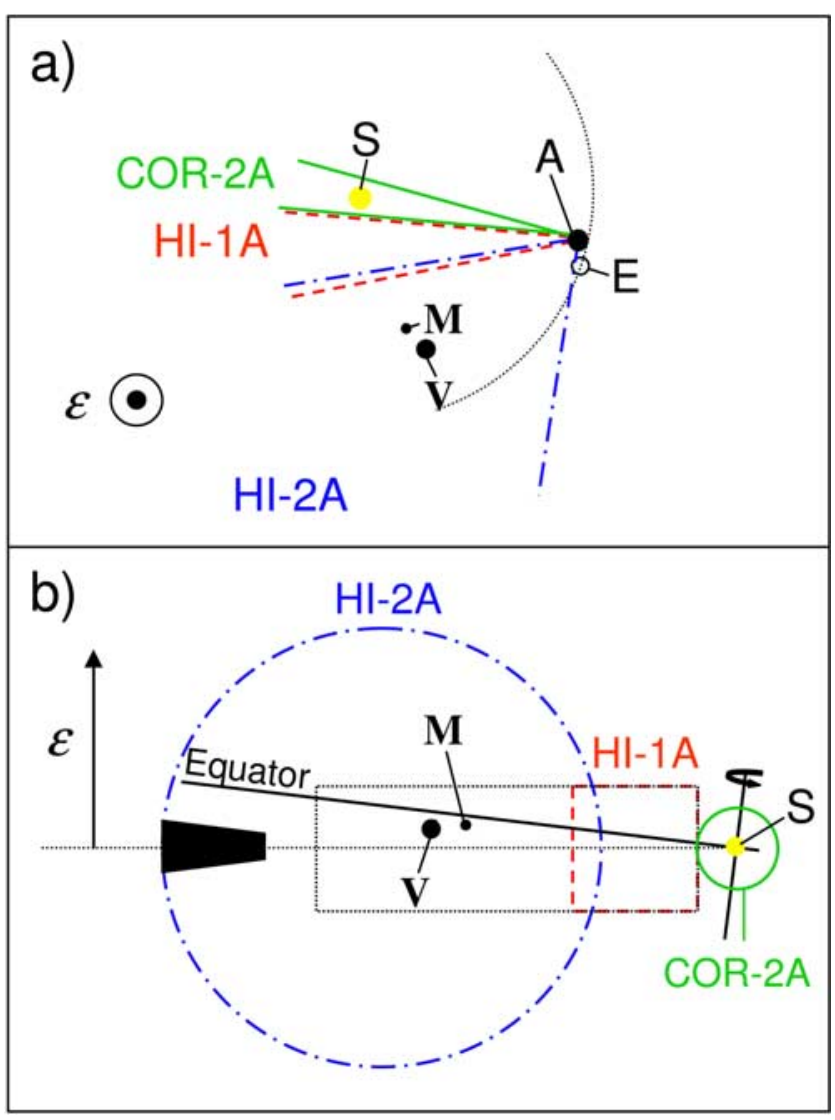

Figure 1. Figure 1a is a view of the ecliptic plane from above showing the position of the Earth (E), Venus (V), MESSENGER (M), and STEREO-A (A). The Sun is the yellow dot labeled $\mathrm{S}$ on the diagram. The limits, in this plane, of the fov of COR-2A (green), HI-1A (red), and HI-2A (blue) are also marked. Vector $\epsilon$ is orthogonal to the solar ecliptic and is located in the solar meridional plane. Figure $1 \mathrm{~b}$ shows the extents of the full fov of COR-2A (green), HI-1A (red), and HI-2A (blue) in the meridional plane. The truncated $\mathrm{HI}$ fov shown in Figures $5 \mathrm{a}, 5 \mathrm{~b}$, and $5 \mathrm{c}$ is marked by a dotted rectangle. The intersection of the solar equator with the solar meridional plane passing through Venus is plotted as a solid black line.

leading to the deformation of B; B becomes highly asymmetric in this COR-2A image and in the $\mathrm{HI}$ images as we will see later. Structure D is a non circular loop in Figure 2d. These white light difference images are derived from line-ofsight integrated photospheric light and the integration of light scattered by two transients propagating along different solar longitudes could give the false impression of interaction.

[11] The emergence of the two transients (A and B) and (C and D), along a similar PA $\left(\sim 90^{\circ}\right)$, is followed by the emergence of smaller events including two more $\mathrm{V}$-shaped structures (features $E$ and $G$ in Figures $2 \mathrm{e}$ and $2 \mathrm{f}$ ) with poorly resolved leading edges, $\mathrm{F}$ and $\mathrm{H}$.

[12] Blobs of plasma were also emitted along the northern footpoint of the main flux rope (indicated by a white arrow in Figure $2 \mathrm{~d}$ at $\mathrm{PA} \sim 45^{\circ}$ ) and along the PA of the central axis of the largest $\mathrm{CME}\left(\sim 90^{\circ}\right)$. Such posteruptive events have often been associated with the formation of an unstable current sheet near the footpoint of the transient and along the central axis of the CME [Lin and Forbes, 2000; Schwenn et al., 2006]. The plasma parcel emitted along the northern footpoint of the main CME, indicated by the white arrow, could be related to the type of interchange reconnection proposed by Crooker and Webb [2006].

\subsection{Defining the Main CME Event}

[13] Recently Wang and Sheeley [2006] showed that the line of sight integration of photospheric light scattered by the plasma distributed on the surface of a flux rope can lead to the formation of $\mathrm{V}$ - or U-shaped structures. It is therefore tempting to relate the presence of $\mathrm{V}$ - or U-shaped structures in the vicinity of large-scale transients to such geometrical effects. When the CME source region can be determined on the photosphere (such as a sunspot, bipolar active region or a filament eruption), the white light appearance of CMEs can also be interpreted in terms of a flux tube orientation dictated by the orientation of the neutral line and/or the overlying prominence [Cremades and Bothmer, 2004] providing additional support to the idea that the flux rope orientation dictates the appearance of the CME in white light.

[14] The structure B at the rear of the main CME event could be the result of a line-of-sight integration effect [Wang and Sheeley, 2006; Thernisien et al., 2006]. The V-shaped structure observed in the COR-2A images was therefore analyzed using the model of Thernisien et al. [2006]. The model is based on a forward modeling technique applied to an empirically defined model of a flux rope, the graduated cylindrical shell (GCS). An electron distribution through the GCS is then assumed to compare with white light coronagraph observations. A synthetic image is derived in terms of total and polarized brightness for various orientations of the model using a Thomson scattering ray-tracing program. The main $\mathrm{CME}$ event was fitted using this technique, as shown in Figure 3. The best fit is obtained when the GCS boundaries are successfully bound by the outer edge of the CME as well as the V-shaped structure. The orientation of the flux rope which matches most successfully the white light images (1) has a longitudinal separation relative to the Sun-STEREO-A line of $\sim 50^{\circ}$, (2) has its footpoints rooted in the northern and southern hemisphere at equal distances from the solar equator, and (3) has a tilt of $\sim 24^{\circ}$ relative to the solar equator. The model confirms that the $\mathrm{V}$ shape of structure B could result from the integration of photospheric light scattered by electrons distributed on a flux rope observed partially edge-on.

\subsection{Latitudinal Distribution of the Storm}

[15] Later in the paper, we will demonstrate that the main CME event impacted the Venus-orbiting Venus Express (VEX) spacecraft as well as the MESSENGER spacecraft. These two spacecraft were located at heliocentric coordinates $\left(\mathrm{r}_{V}, \phi_{V}, \theta_{V}\right)=\left(0.72 \mathrm{AU}, 305.4^{\circ},-3.51^{\circ}\right)$ and $\left(\mathrm{r}_{M}, \phi_{M}\right.$, $\left.\theta_{M}\right)=\left(0.66 \mathrm{AU}, 304.4^{\circ},-1.6^{\circ}\right)$, respectively, at the time of impact. The PAs of MESSENGER and VEX were calculated to be $91.3^{\circ}$ and $93.7^{\circ}$, respectively. The radial evolution of CMEs can be characterized using the J-mapping technique of Sheeley et al. [1997]. To determine the parts of the solar storm likely to intersect the orbits of MESSENGER and VEX, we created COR-2A J maps along these two PAs rounded to the nearest integer value $\left(91^{\circ}\right.$ and 

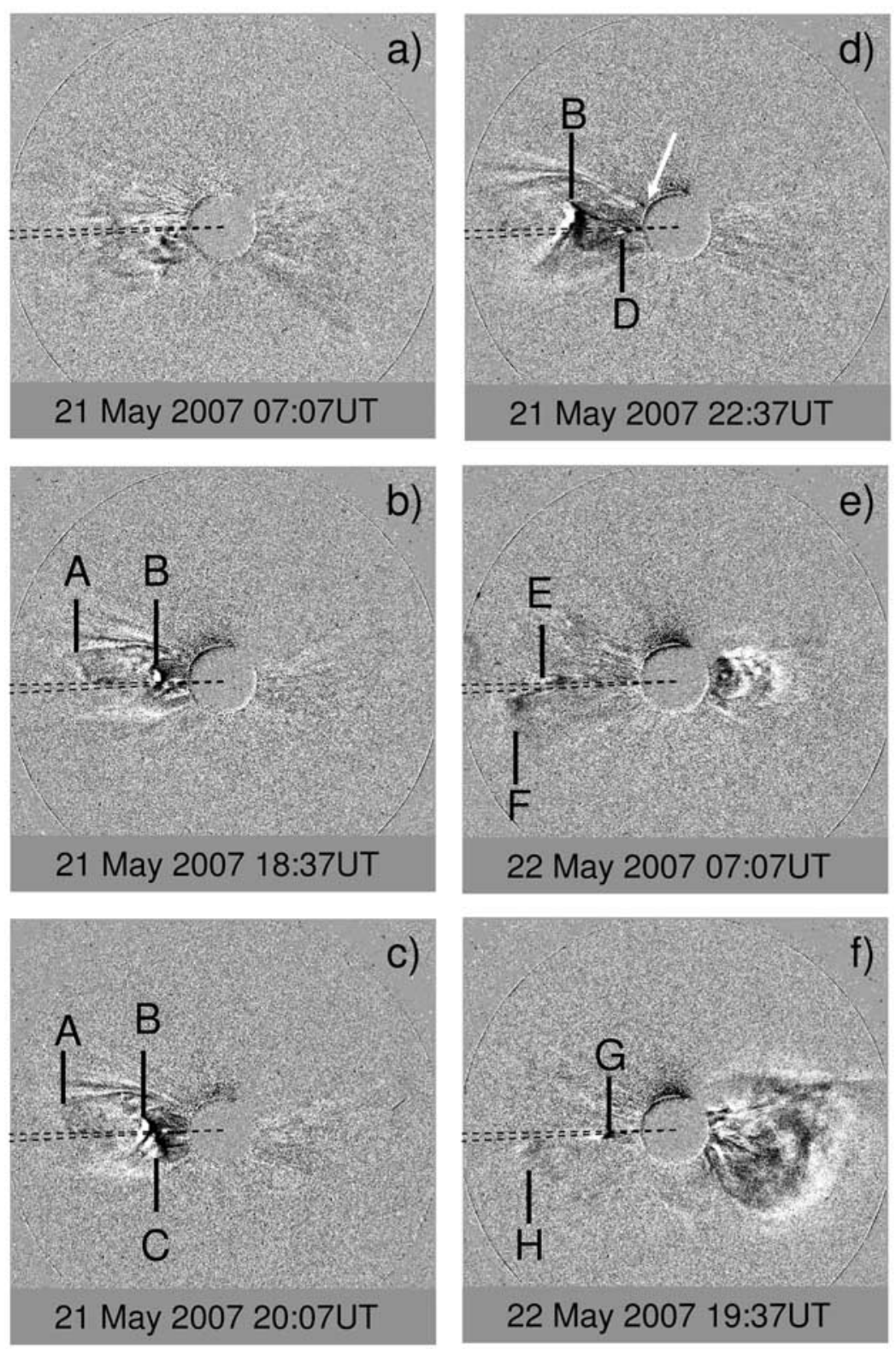

Figure 2. A series of six COR-2A running difference images presented in chronological order showing the emergence of the solar storm from the East Limb of the Sun (i.e., to the left). A total of eight features associated with different coronal transients are identified and are labeled from A to $\mathrm{H}$. A white arrow in Figure $2 \mathrm{~d}$ marks the location a plasma blob takeoff at the northern footpoint of the main CME. The solar radials along which MESSENGER and VEX are located are marked by dotted lines at PA $\sim 91^{\circ}$ and $\mathrm{PA} \sim 94^{\circ}$, respectively.

$94^{\circ}$, respectively). This mapping technique involves the creation of a time-height map, constructed by stacking a time series of radial profiles (i.e., along a constant PA) extracted from a sequence of coronagraph images. We show these $\mathrm{J}$ maps in Figure 4, as well as a third $\mathrm{J}$ map along PA $=100^{\circ}$ where many transients are observed to emerge. The tracks made by the various transients imaged in Figure 2 are labeled accordingly in Figure 4. Only one distinct track is visible along $\mathrm{PA}=91^{\circ}$, whereas many tracks are observed in the other two J maps. Loop C, which seems to collide with the main CME, appears more clearly along $94^{\circ}$ and $100^{\circ}$, and, indeed, in these latter J maps the main CME seems to merge with structure $\mathrm{C}$. Tracks $\mathrm{E}$ and $\mathrm{F}$, which are related to the emergence of subsequent loops and V-shaped structures, are also absent along $\mathrm{PA}=91^{\circ}$. Although $\mathrm{G}$ is a significant feature at $\mathrm{PA}=100^{\circ}$, it is totally absent at $91^{\circ}$ and leaves only a very faint signature at $94^{\circ}$.

[16] The COR-2A and COR-2B instruments were not separated enough to allow 3-D reconstruction nor trajectory estimates at the time. However, from the COR-2A data alone we can determine at least in terms of latitude which parts of the solar storm could have impacted the VEX and MESSENGER spacecraft. If we assume longitudinal alignment, we can make the following prediction based on the COR-2A observations: 


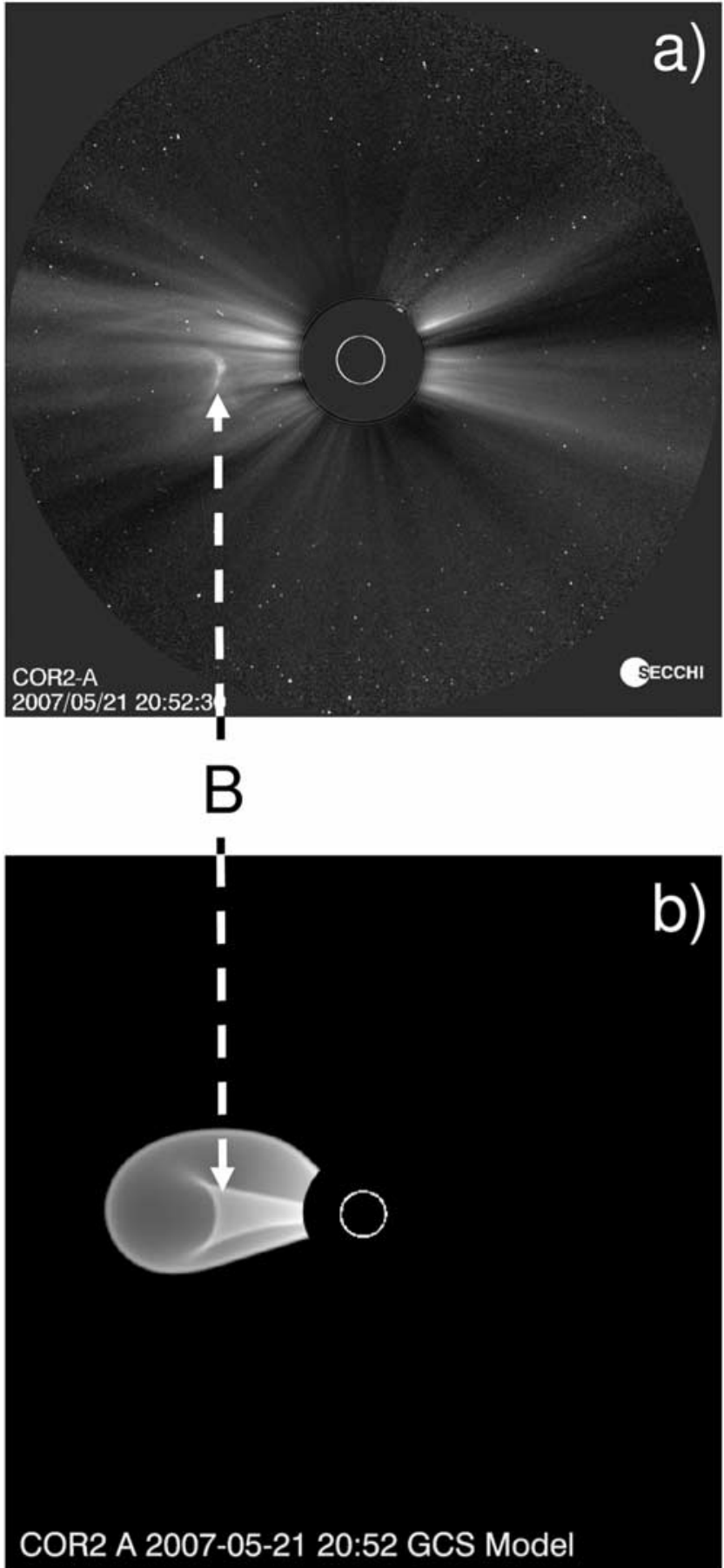

Figure 3. (a) A COR-2A background subtracted image taken at 2052 UT on 21 May 2007 during the main CME takeoff. (b) The simulated white light appearance by the method of Thernisien et al. [2006]. The leading edge and $\mathrm{V}$-shaped structure appear clearly in that simulation.

[17] 1. MESSENGER and VEX should both detect a large ICME event (structure A and B) with two broad density enhancements bounding a large-scale flux rope.

[18] 2. MESSENGER may also be intersected by a second smaller CME (structure $\mathrm{C}$ and D). However, VEX was better located in latitude to intersect the structure as well as subsequent features $\mathrm{E}$ and $\mathrm{F}$.
[19] 3. Neither MESSENGER nor VEX could have been hit by the transient $\mathrm{H}$ which had a central axis located at $\mathrm{PA} \sim 100^{\circ}$.

\subsection{HI Observations of the CME}

[20] The edges of the CME, initially observed by COR2A, are also seen in HI images from STEREO-A (Figures $5 \mathrm{a}, 5 \mathrm{~b}$, and $5 \mathrm{c})$, propagating out from the Sun through the inner HI-1A and subsequently the outer HI-2A fovs (i.e., right to left). The HI-1A and HI-2A images are running difference images. Not only does this minimize the contribution of the stable $\mathrm{F}$ corona, but it is a very useful technique for highlighting faint propagating features as already demonstrated with the COR-2A images. CME feature A, identified as a loop in the COR-2A images, appears in HI as a half loop while feature B has retained its curved (nearly V-shaped) aspect.

[21] Although the brightness of features A and B is very faint when they reach the middle of the HI-2A fov, the two plasma elements appear to sweep over Venus, apparent in the HI-2A images at $(\alpha=) 46^{\circ}$ elongation. This could simply be a result of perspective; the longitude $(\beta)$ of propagation of the CME could be such that it actually passes behind or in front of the planet. At the time of CME launch, the longitude difference between Venus and STEREO-A (Venus-SunSTEREO-A angle) was $\beta \sim 60^{\circ}$.

[22] The line of pixels along the PA (angle from solar north) of Venus $\left(94^{\circ}\right)$ is extracted from each HI-1 and 2A running difference taken between 21 and 27 May 2007, and used to construct the $\mathrm{J}$ map shown in Figure 6. This technique is presented in full detail by Davies et al. [2009]. Three large tracks emerge between 21 and 23 May 2007. The first track corresponds to a small event which emerged before the main CME and disappeared quickly as it progressed outward; this quick fading suggests that this event propagated from the East limb of the Sun, away from the Thomson sphere. The second and third tracks correspond to features $\mathrm{A}$ and $\mathrm{B}$ identified in COR-1/2A and HI-1/2A images. The elongation angle of track A changes more rapidly with time than the elongation angle of track $\mathrm{B}$, this suggests that feature A is moving faster. Sheeley et al. [2008a, 2008b] and Rouillard et al. [2008] have highlighted the importance of the longitude separation between the observer (here STEREO-A) and the radially out-flowing transient in dictating the slope and shape of the CME/CIR tracks observed in $\mathrm{J}$ maps. We extracted the elongation variation of tracks A and B by selecting points on the white to black boundary seen on the $\mathrm{J}$ maps. This boundary is located to the rear of the actual plasma parcel seen in background subtracted images.

[23] The elongation, labeled $\alpha$ in Figure 7, of a point $\mathrm{P}$ in the solar wind observed by the STEREO-A spacecraft is defined as the Sun-STEREO-A-P angle, being zero at Sun centre. The angular separation between the Sun-STEREOA spacecraft line and the direction of propagation of the point $\mathrm{P}$ (labeled $\beta$ in Figure 7 ) equates to the longitude separation in an ecliptic based heliocentric coordinate system $\left(\phi_{C M E}-\phi_{A}\right)$ when the transient propagates in the ecliptic plane. The elongation variation, $\alpha(t)$, of a solar wind transient depends upon its radial speed, $V_{r}$, and the angle $\beta$ [Rouillard et al., 2008, 2009]. Best fit values of these parameters can, therefore, be extracted from the $\alpha$ 
2007-5-21 to $2007-5-22$
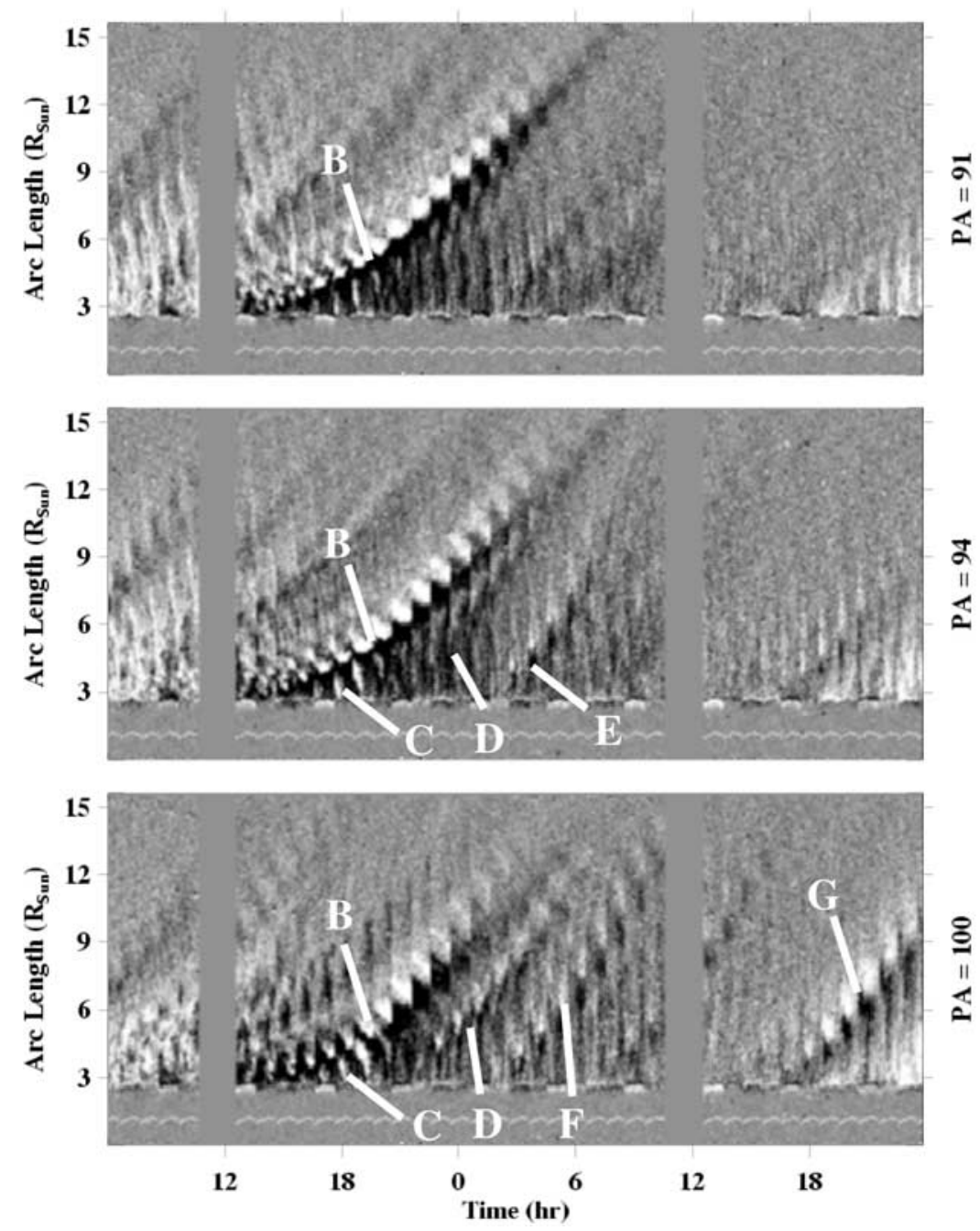

Figure 4. Three COR-2A J maps along the PA of MESSENGER $\left(91^{\circ}\right)$, along the Pa of Venus $\left(94^{\circ}\right)$, and at $\mathrm{PA}=100^{\circ}$. The tracks of the various transients identified in COR-2A running difference images are labeled in these J maps accordingly. Arc Length $\left(\mathrm{R}_{\text {Sun }}\right)$ is the parameter used traditionally on the $\mathrm{Y}$ axis of $\mathrm{J}$ maps derived from coronagraph observations. We substitute this with elongation angle $\alpha$ in the HI $\mathbf{J}$ map in Figure 6.

elongation variation recorded by HI [Rouillard et al., 2008; Sheeley et al., 2008a, 2008b]. Applying the technique of Rouillard et al. [2008, 2009] to the elongation variations of features A and B as observed by HI, produces best fit values for the speed $V_{r}$ and the longitude separation angle $\beta$ of

$$
\begin{gathered}
V_{A}=333 \pm 4 \mathrm{~km} \mathrm{~s}^{-1}, \beta_{A}=60 \pm 11^{\circ}, \\
V_{B}=305 \pm 20 \mathrm{~km} \mathrm{~s}^{-1}, \beta_{B}=72 \pm 18^{\circ} .
\end{gathered}
$$

The uncertainties are calculated from the statistical scatter of the chosen points about the best fit.

[24] The points selected on the $\mathrm{J}$ maps are shown in Figure 8 and the fit plotted as a solid black line. The uncertainty in the estimated speeds is small compared to the uncertainty in the estimated directions. The large uncertainties in the estimate of $\beta$ are related to the data gaps which affect the quality of the $\mathrm{J}$ maps for that particular event. The CME is also propagating away from the Thomson sphere and hence its signal drops very quickly in HI-2 images. We note, however, that a major source of uncertainty in the $\mathrm{J}$ map fitting technique is caused by the (human) choice of the main CME track on the J map. Repeating the analysis many times, we find that variations of $30-40 \mathrm{~km} \mathrm{~s}^{-1}$ occur in the estimated speed, and $4-5^{\circ}$ in $\beta$ angles. A more continuous tracking of the CME would have resulted in a more accurate fit.

[25] Nevertheless, as the longitudes of propagation of the two fronts agree within errors, we assume an average value $\left(66^{\circ}\right)$; this single trajectory is plotted in Figure 7 as a black arrow. The latitudinal extents of A and B are approximately $35^{\circ}$ and $30^{\circ}$, respectively. Cane et al. [1997] noted that remarkably few ICMEs have been observed by pairs of spacecraft even separated by only $40^{\circ}$ in longitude and concluded that ICMEs typically extend over $\sim 50^{\circ}$ in longitude, similar to the average latitudinal extent of CMEs 
a) 12 UT, 22 May 2007

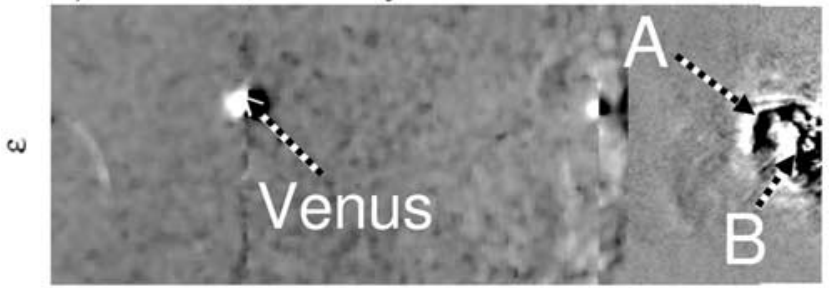

b) 18 UT, 22 May 2007

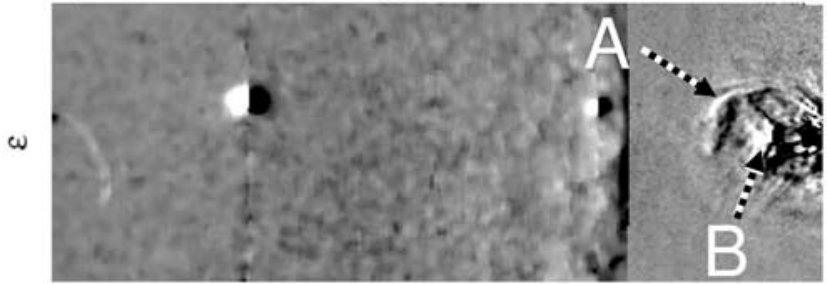

c) 00 UT, 24 May 2007

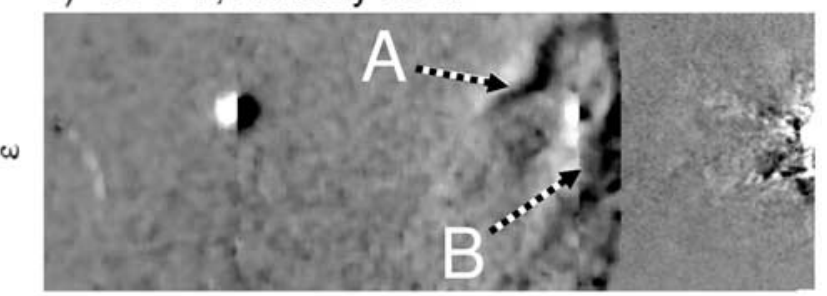

60

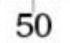

40

30

20

10

Figure 5. Three HI-1/2A composite images consisting of HI-1A and HI-2A running difference images. The fields of view were truncated by the rectangular limit shown in Figure 1a. Structures A and B identified in the COR-2A images are also labeled here. The elongation angle $(\alpha)$ is that of the central row and is the angular distance from Sun center. Venus is the bright white and black feature at an elongation angle of $\alpha=46^{\circ}$.

observed in coronagraphs [St. Cyr et al., 2000]. We have no direct measure of the longitudinal extent of this main CME but it is likely that it exceeds $12^{\circ}$ in half width. Thus the proximity in longitude to Venus's location is strongly suggestive of CME-planetary impact. The impact times of fronts $\mathrm{A}$ and $\mathrm{B}$ at Venus, derived from the fitted parameters for the elongation, $\alpha=46^{\circ}$ of the planet, would be near 0900 UT on 25 May and 0900 UT on 26 May. The fit, however, was evaluated using points selected on the white to black boundary on these $\mathrm{J}$ maps. The boundary between the black and white areas marks, in these $\mathrm{J}$ maps derived from difference images, where the brightness increases and decreases because of the passing plasma elements. The local density increases during the passage of plasma elements A and $\mathrm{B}$, however, are located inside the white area (regions of increasing brightness). The width of the white region is evaluated in the near-Sun portion of the J map (inner edge of HI-1A) when the features are best observed. This white region is used to derive the width of the density increase. A simple inspection of the $\mathrm{J}$ maps shows that the density increase associated with feature $\mathrm{A}$ has a width of $5 \mathrm{~h}$ at $\alpha=$ $5^{\circ}$ while feature B extends over a time width of $7 \mathrm{~h}$ at $\alpha=$ $5^{\circ}$. The predicted arrival times of the leading edge of each plasma parcel should therefore be the predicted arrival times derived from the fit minus the 5 and $7 \mathrm{~h}$, respectively:

$$
t(A)=25 \text { May } 20070400 \mathrm{UT}
$$

$$
t(B)=26 \text { May } 20070200 \text { UT }
$$

Assuming the trajectories and transient speeds are correctly predicted, these are the predicted arrival times of the leading edges of features $\mathrm{A}$ and $\mathrm{B}$ at Venus using the J-mapping technique.

[26] The source region of the main CME event could be located by using the estimated trajectory. The CME originated on the western edge of a large equatorial coronal hole. Large equatorial coronal holes are prone to force the formation of CIRs in the interplanetary medium because they tend to emit fast solar wind at the same latitude as the streamer belt. As we shall show using in situ data the CME was propagating in a rarefaction region higher up in the corona.

[27] The HI cameras have removed ambiguities concerning the longitude of propagation of the main CME event (comprising features $\mathrm{A}$ and $\mathrm{B}$ ). We conclude the following from the HI images:

[28] 1. MESSENGER and VEX should have been hit by a large CME event 3.46 and 3.7 days after CME launch (time of propagation for an average transient speed $\left(\sim 317 \mathrm{~km} \mathrm{~s}^{-1}\right)$.

[29] 2. This CME, which originated near the eastern boundary of a coronal hole, almost certainly propagating in a rarefaction region located in the trailing edge of a CIR.

[30] The elongation fitting technique suggests that the leading edge of the transient was moving faster than its trailing edge and, as we shall see in section 5, in situ observations confirm this is the case.

\section{In Situ Observations of the CME \\ 4.1. Venus Express}

\subsubsection{ICME Passage at Venus}

[31] The Venus Express (VEX) spacecraft is in a 24-h elliptical near-polar inertially fixed orbit around Venus with pericenter and apocenter around $250 \mathrm{~km}$ and $60000 \mathrm{~km}$ altitude, respectively. VEX carries a magnetometer which operates almost continuously [Zhang et al., 2006] and a plasma analyzer, ASPERA-4 (Analyzer of Space Plasmas and Energetic Atoms) [Barabash et al., 2007]. The latter detects ions, electrons and energetic neutral atoms, to enable study of the near Venus environment [Coates et al., 2008], and usually operates only when inside Venus's magnetosheath, starting and ending just outside the Venusian bow shock. ASPERA-4 does, however, occasionally take measurements around satellite apocenter, as is the case during the period presented here. VEX spends about $21 \mathrm{~h}$ of its $24 \mathrm{~h}$ orbit outside the induced 


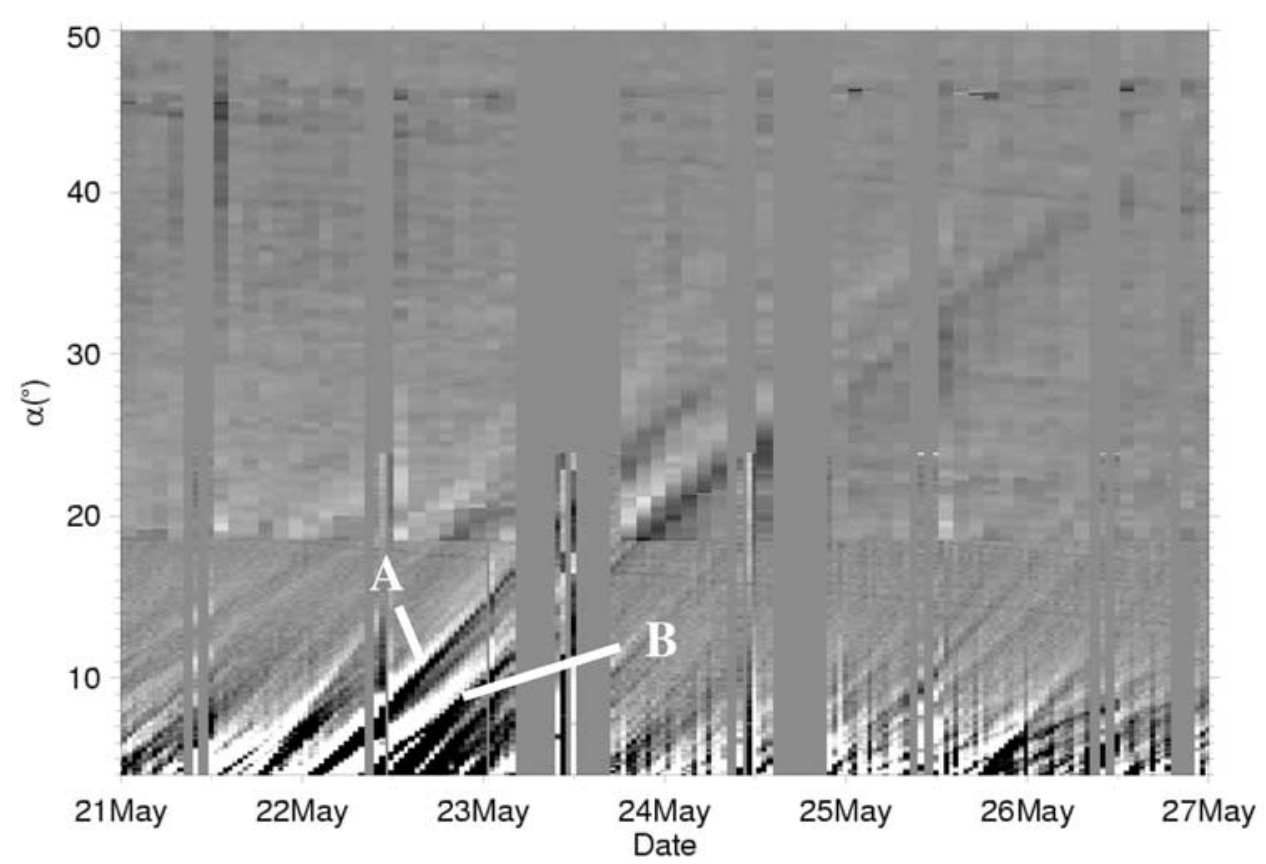

Figure 6. A J map obtained from HI-1/2A difference images tracking the outward propagation of the main event (features A and B are labeled). The strips of images affected by missing data or bad background subtraction are masked in light grey.

magnetosphere of Venus, and can thus be used to study the structure of the solar wind and the embedded interplanetary magnetic field (IMF) at 0.72 AU.

[32] The magnetic field azimuth angle, elevation angle and strength from VEX are shown in RTN (Radial, Tangential, Normal) coordinates in Figures $9 \mathrm{a}, 9 \mathrm{~b}$, and $9 \mathrm{c}$, respectively. The magnetosphere crossings have been removed from the data sets; therefore all time series shown in this plot are pure solar wind measurements taken in the vicinity of Venus. The solar wind density and speed, taken near apocenter by the ion experiment on ASPERA-4, are shown in Figures 9d and 9e as black points. The heliocentric latitudes of VEX and ACE are plotted in Figure 9f as a dotted and black line, respectively. VEX and ACE recorded the solar wind plasma at different latitudes throughout the interval.

[33] We compared the observed solar wind stream profile measured at Venus to the one observed a few days later at Earth to test the accuracy of the calibration of ASPERA data. The ACE (near 1AU) data was mapped ballistically to $0.72 \mathrm{AU}$ by assuming that each quiet solar wind parcel moves at a constant speed. The time taken for coronal holes to rotate from the longitude of Venus to that of Earth was obtained by using the time-varying longitude separation between Venus and Earth divided by the angular rotation rate of the corona near the equatorial regions $\left(2.7910^{-6} \mathrm{rad}\right.$ $\left.\mathrm{s}^{-1}\right)$. The ACE solar wind density and speed measurements mapped to Venus are plotted as black lines in Figures 9d and 9e. The good correlation between the two sets of observations of the solar wind speed is clear in Figure 9e, in particular between 20 May and 24 May. The similarity between the solar wind speed observed by ACE and at Venus suggests that there was little evolution of the coronal holes during the interval of time separating VEX and ACE measurements and, at these low latitudes there was little latitudinal variation in the emitted solar wind speed.

[34] The densities are similar until 24 May 2007 and differ significantly thereafter. In the interval during which the data sets show a good correspondence, between 20 and 24 May, the density is low and the IMF magnitude gradually declines. During this period, the IMF undergoes large

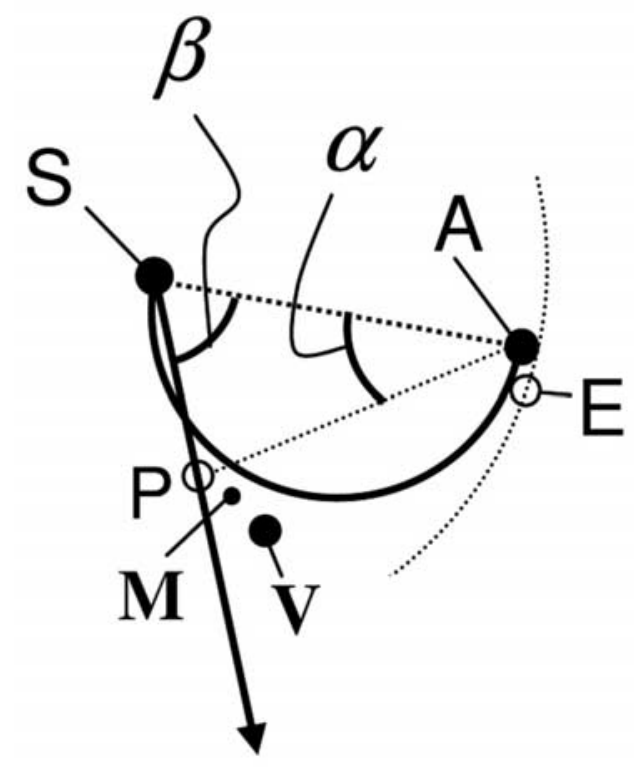

Figure 7. View of the ecliptic plane from above in the same format as Figure 1a. The average CME path estimated from the elongation variations of $\mathrm{A}$ and $\mathrm{B}$ is marked by a black vector. The longitude $(\beta)$ of the CME trajectory and the elongation $(\alpha)$ of a point $\mathrm{P}$ on its trajectory are shown. 


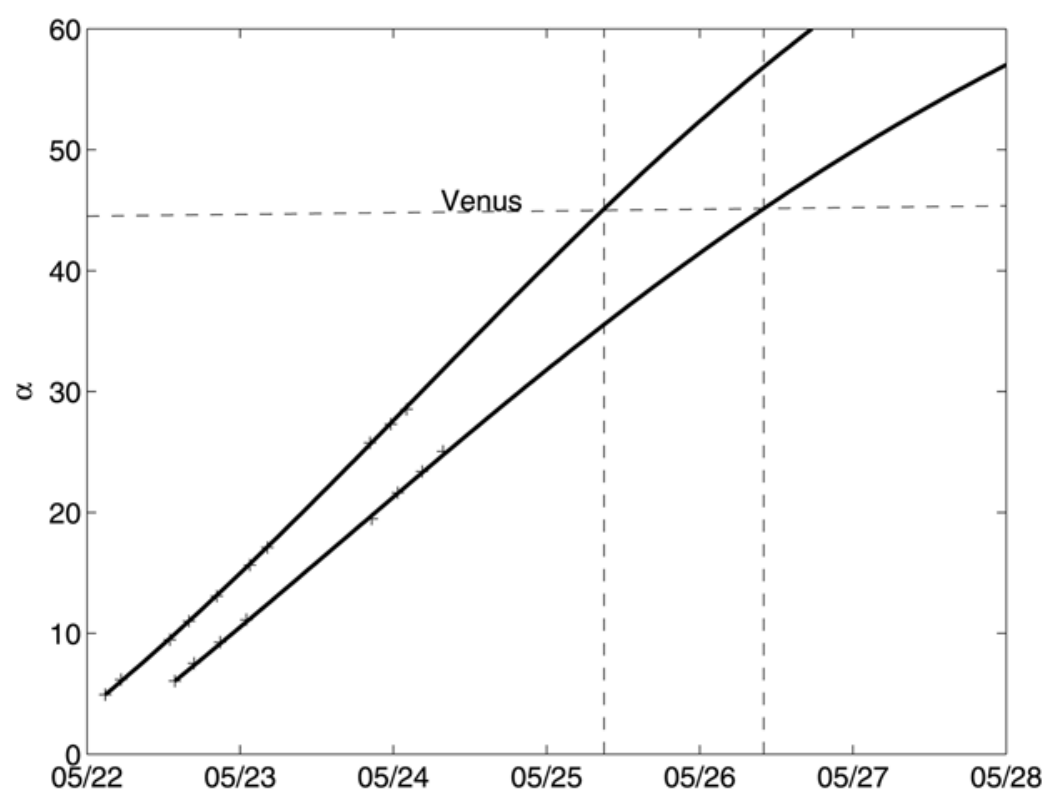

Figure 8. The points selected on the J maps (black-white boundary) and the best fit estimate using the method of Rouillard et al. [2008]. The elongation of Venus is plotted by a horizontal dotted line. The times of intersection of the two fitted lines with Venus's elongation are marked with two vertical lines and define the arrival times of the black-white boundaries of the features seen in the J maps (i.e., the rear parts of features $\mathrm{A}$ and $\mathrm{B}$ ).

high-frequency variations in direction, characteristic of the fast solar wind [Belcher and Davis, 1971]. This evolution of the solar wind parameters is typical of a rarefaction region, formed where fast solar wind $\left(>600 \mathrm{~km} \mathrm{~s}^{-1}\right)$ is emitted from the Sun ahead of slow solar wind (typically $<400 \mathrm{~km} \mathrm{~s}^{-1}$ ). The ACE and ASPERA-4 measurements confirm that a decrease in solar wind speed occurred during this interval. A systematic decrease in the magnetosheath field strength is also observed (not shown here) between 22 and 24 May and is a natural response of the Venus induced magnetosphere to the rarefaction in the solar wind.

[35] From about 2000 UT on 24 May until the end of 26 May, the IMF increases in magnitude (Figure 9c) and this is immediately followed by a large-scale rotation in the field directions (Figures 9a and 9b). These are both common characteristics of flux ropes recorded in situ by spacecraft in interplanetary space [Burlaga, 1988; Rees and Forsyth, 2004] and, hence, provide clear evidence of an ICME crossing the VEX orbit at that time. The arrival of this flux rope is correlated in time with a sudden change in solar wind ion density measured by VEX (Figure 9d) and marks the onset of poor correlation between the ACE and VEX observations of solar wind density. We suggest that this sudden discrepancy is induced by the arrival of the ICME plasma which was observed at Venus Express but not at ACE. The predicted arrival times of the white light features $\mathrm{A}$ and $\mathrm{B}$ of the CME (seen in Figures 2 and 5) are shown as vertical black lines in all plots. The sudden increase in ion density detected by ASPERA-4 between 24 and 26 May (Figure 9d) coincides with the predicted arrival time of the two density fronts tracked by STEREO. The leading edge of feature A arrives with the onset of the flux rope rotation while the leading edge of the V-shaped structure (feature B) arrives as the spacecraft leaves this flux rope. Features A and B therefore bound the limits of the main flux rope and their presence explain the sudden increase in ion density during this period.

[36] In a crude attempt to estimate the in situ speed of each feature, we have interpolated linearly the ASPERA-4 data sets to obtain an estimated in situ measurement of the speed of features A and B; these speeds are found to be $385 \mathrm{~km} \mathrm{~s}^{-1}$ and $355 \mathrm{~km} \mathrm{~s}^{-1}$, respectively. These values are similar to the speeds obtained from the elongation imprint of the transients in HI $\left(333 \mathrm{~km} \mathrm{~s}^{-1}\right.$ and $\left.305 \mathrm{~km} \mathrm{~s}^{-1}\right)$. We note that for both of these events the J-mapping technique predicts speeds that are $50 \mathrm{~km} \mathrm{~s}^{-1}$ too slow; this could be the result of the poor quality of the $\mathrm{J}$ maps for this particular event. The predicted arrival time from the $\mathrm{J}$ map fitting technique was based on speeds which are on average $\sim 50 \mathrm{~km} \mathrm{~s}^{-1}$ too slow. At an average predicted speed of $320 \mathrm{~km} \mathrm{~s}^{-1}$ an increase of about $50 \mathrm{~km} \mathrm{~s}^{-1}$ will decrease the transit time from the Sun to $0.72 \mathrm{AU}$ by about $11 \mathrm{~h}$. The arrows in Figure 9d mark the systematic shift that should be applied to the estimated arrival time of A and B according to in situ observations. A probably arrived directly ahead and outside the flux rope rotation and B occurs right at the end of the flux rope rotation in Figure 9. This is supported directly by the ASPERA-4 density measurements which increase dramatically already $10 \mathrm{~h}$ before the main flux rope rotation.

\subsubsection{Topology of the Magnetic Field}

[37] The magnetic field elevation angles measured by VEX reveal a succession of distinct large-scale variations. The sparsity of the plasma data means we rely mostly on analyzing the magnetic field data. Different structures are identified from elevation versus azimuth plots of the magnetic field orientation $(\theta-\phi$ plots). This technique has been used in the past to study the degree of organization in the 


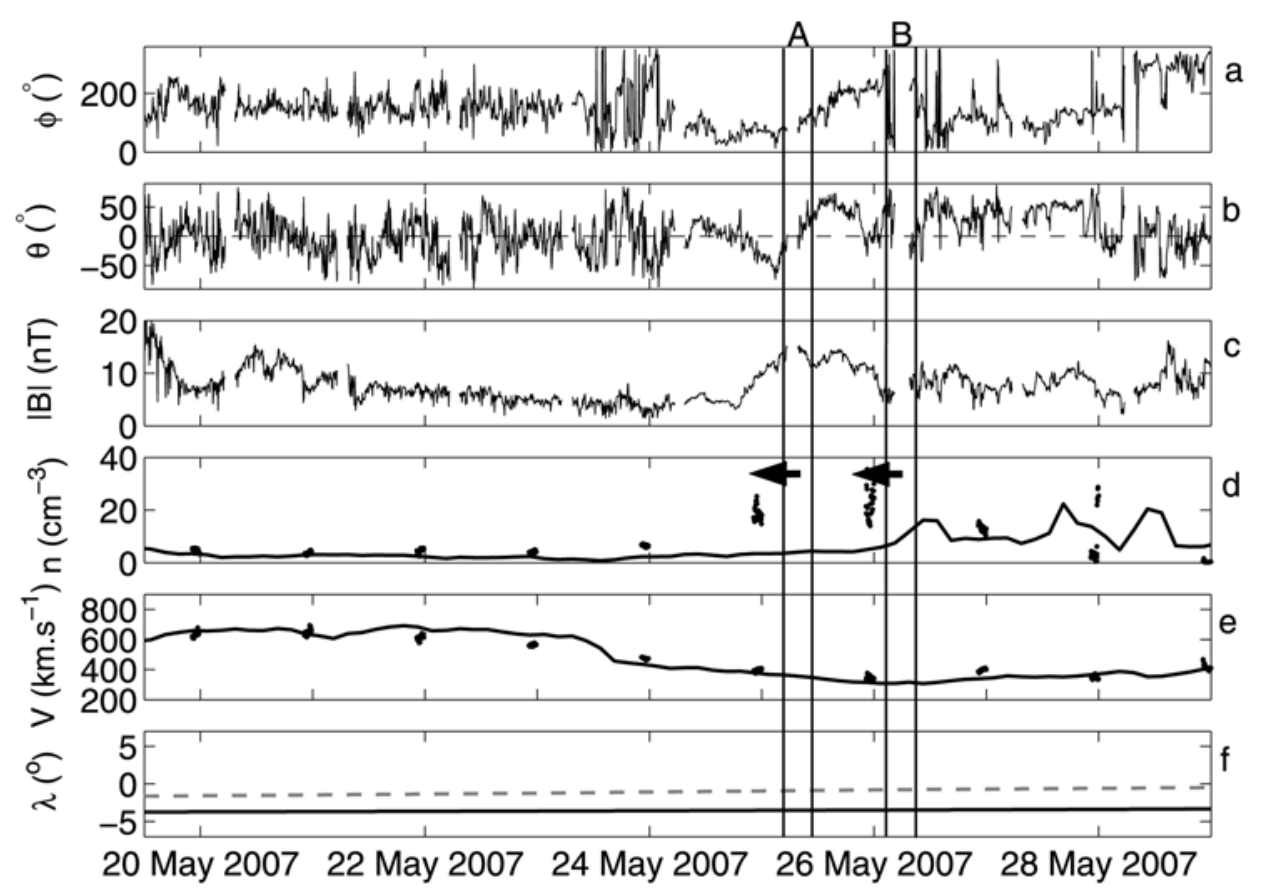

Figure 9. The azimuth angle, elevation angle and strength of the magnetic field from the VEX magnetometer are plotted in Figures 9a, 9b, and 9c. The VEX ASPERA-4 measurements of the density and speed of the solar wind ions are plotted as dots in Figures 9d and 9e, respectively. The estimated density and speed of the solar wind at VEX inferred by mapping the ACE data to VEX are plotted as a black line in Figures 9d and 9e. The latitudes of Venus Express and Earth during these measurements are plotted as a dotted line and a black line, respectively, in Figure 9f. The times of arrival of features A and $\mathrm{B}$ are marked by two pairs of vertical lines crossing the five plots; each pair marks the leading and rear edge of each structure as determined by white light observations.

magnetic field and to assess if the field deviates from the Parker spiral (i.e., deviating from the orientations; inward field $\left(\phi=145^{\circ}, \theta=0^{\circ}\right)$, outward field $\left(\phi=325^{\circ}, \theta=0^{\circ}\right)$ at Venus) [Nakagawa et al., 1989; Farrugia et al., 1990; Neugebauer et al., 1993; Crooker et al., 1993]. We present this analysis in RTN coordinates in Figure 10. Magnetic fields (Figure 10a) with mean azimuth, $\phi=61^{\circ}$, and mean elevation, $\bar{\theta}=-1.8^{\circ}$ (i.e., located in the RT plane) and therefore distributed across the nominal spiral field orientation are observed ahead of a full rotation of the magnetic field (Figure 10b). Subsequently, two distinct ordered structures (Figures 10c and 10d) are observed, followed by a long period $(24 \mathrm{~h})$ of highly elevated magnetic field (pointing northward: $\bar{\theta}=41^{\circ}$ ) (Figure 10e). A current sheet crossing is also observed a day later with the arrival of a CIR and its associated compressed magnetic fields (Figure 10f).

[38] The magnetic field in Figures 10b, 10c, 10d, and 10f mark successive periods of different wave-like patterns which are significant deviations from the typical Parker spiral orientation. The intervals of time corresponding to each plot are marked by different shades of grey in Figures 11a, 11b, and 11c which shows a comparison of VEX and MESSENGER magnetic field direction and strength measurements. On scales of seconds to hours, most of the features observed in the IMF, such as shocks, discontinuities and Alfvén waves are locally planar structures because their thicknesses or wavelengths are much smaller than their scale sizes which are considerable frac- tions of an astronomical unit. At high temporal resolution, most of these structures as well as magnetic flux ropes can present these partial wave-like patterns on $(\theta-\phi)$ plots.

[39] These wave-like patterns are also similar to the Planar Magnetic Structures (PMS) presented by Nakagawa et al. [1989]. To differentiate PMSs from Alfvén waves or flux ropes, Nakagawa et al. [1989] classified planar magnetic structures as regions of the solar wind where magnetic field orientations are constrained to planes, the plasma $\beta$ is high because of the high prevailing densities and the magnetic field changes direction frequently and abruptly (often driven by tangential discontinuities). None of the wave-like patterns presented in the present study fall in the strict definition of PMSs set by Nakagawa et al. [1989]. The successive intervals identified in Figures 10b, 10c, and $10 \mathrm{~d}$ show that the magnetic field variations were longlasting, large-scale rotations of the magnetic field. These variations differ from the Nakagawa et al. [1989] structures because they are not highly fluctuating variations in the magnetic field.

[40] These large-scale rotations are usually associated with the passage of flux ropes. These flux ropes can acquire a force-free field topology above the photosphere. A forcefree magnetic field, representing a circular cylindrical flux rope (Lundquist solution) similar to that used by Lepping et al. [1990], was therefore used to analyze the magnetic field in Figures 10b, 10c, and 10d. We note that every $24 \mathrm{~h}$ VEX plunged into the magnetosphere for $3 \mathrm{~h}$, the magnetic field 

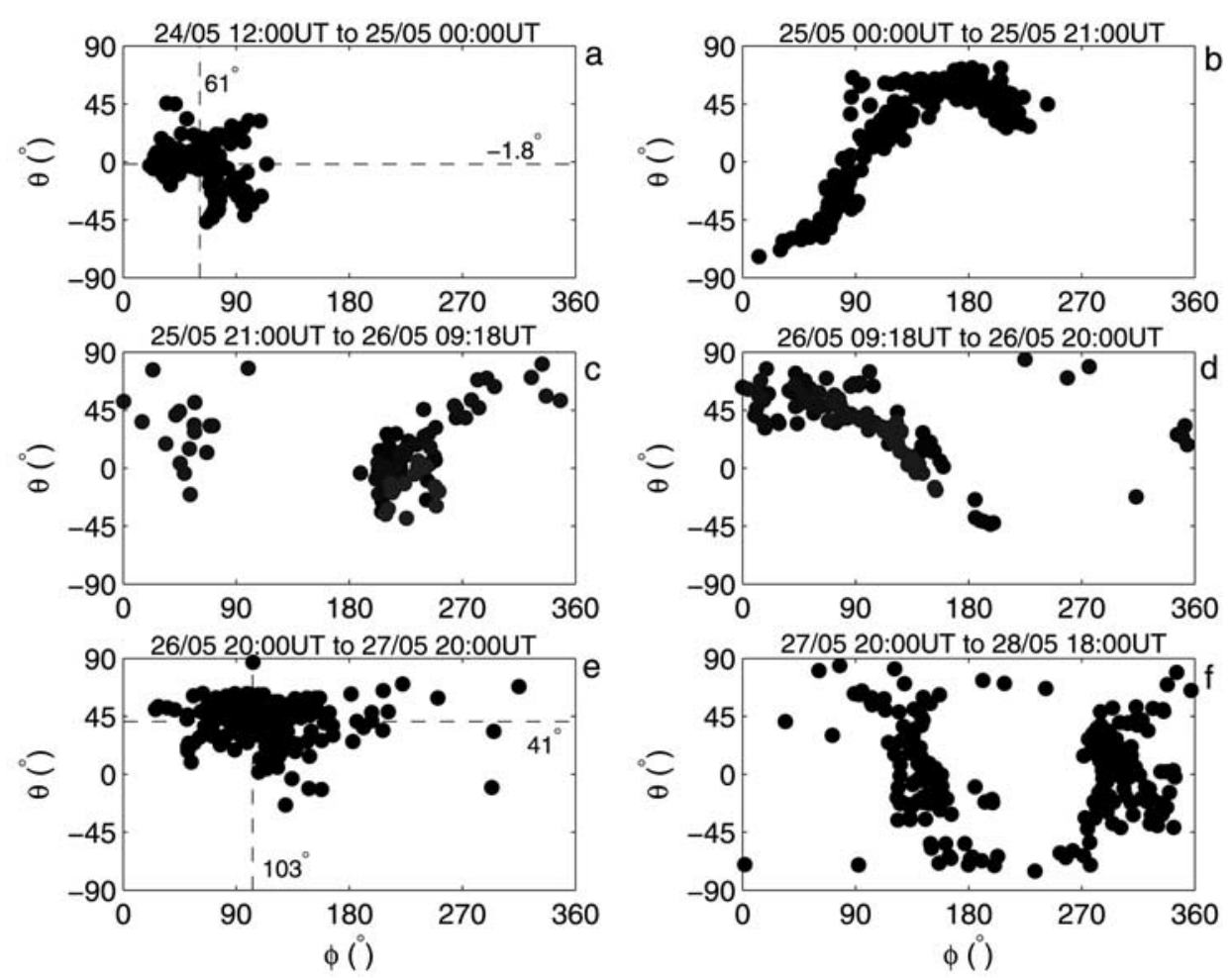

Figure 10. Six intervals of distinct magnetic field variations measured by VEX and determined from $(\phi$, $\theta$ ) plots of the magnetic field orientation. Figures 10a to 10e mark the passage of significantly nonParkerian magnetic fields where the relation between azimuth and elevation forms wave-like patterns (Figures 10b, 10c, 10d, and 10f) or where the average azimuth and elevation of the magnetic field deviates from ideal Parker spiral field orientations (Figures 10a and 10e); the averages of the azimuth and elevation angles are shown by vertical and horizontal dotted lines, respectively, in Figures 10a and 10e.

data during these intervals was therefore ignored in fitting the rope.

[41] Interestingly, the magnetic field enhancement associated with the largest flux rope has a clear double-humped structure which could suggest the main event consisted of two smaller events rather than one single large flux rope. Two arrows in Figures 11c and $11 \mathrm{f}$ indicate the location of these two enhancements. Wang et al. [2002] presented an example of a system of flux ropes passing Earth on 27 November 2000. They found that four flux ropes had to be convolved together to describe the magnetic field variations seen in situ. The lack of plasma composition data and of continuous plasma moment measurements means that we rely exclusively on the magnetic field strength profile to assess the possibility of two independent structures inside this large event, which is not satisfactory. We importantly note, however, that the continuity of the variations of the $\phi$ and $\theta$ angles is suggestive of a single large flux rope.

[42] Nevertheless we attempted two series of fits, (1) assuming a double flux rope and (2) assuming a single large flux rope. In case 1 , while the second rotation of the field could be fitted successfully, the first part of the magnetic field enhancement could not be fitted adequately and we conclude that it is unlikely to be a flux rope. In case 2 , a good fit of the $\phi$ and $\theta$ variations was obtained over the entire event with a cylindrical axis orientation in RTN coordinates given by $[-0.02,0.67,0.74]$. This structure, located between white light features $\mathrm{A}$ and $\mathrm{B}$, is therefore associated with the expected flux rope of the main ICME. The fitted red lines are shown in Figures 11a, 11b, and 11c. Clearly the double increase in the magnetic field strength is not modeled by this single rope, unlike the $\phi$ and $\theta$ variation which are well reproduced. We have found a strong dependence of the fitted rope orientation on the interval of data selected and therefore the derived flux rope axis orientation is tentative.

[43] It was instructive to investigate the nature of the other two wave-like patterns seen in Figures 10c and 10d. The variation seen in Figure 10c could not be fitted by a flux rope model as the magnetic field strength decreases inside the structure while a typical force-free field distribution is such that an increase in the magnetic field strength should occur toward the center of the rope [Burlaga, 1988]. The $\phi$ and $\theta$ angles, however, show a clear smooth rotation during this interval (Figures 11a and 11b) which confirms that the field is highly ordered, and could well be an even smaller scale flux rope with much weaker magnetic field. The proximity of the large CME event ahead of this rope leads to a decrease of the magnetic field strength as the spacecraft leaves the main ICME to enter the smaller-scale event. A model such as the one employed by Wang et al. [2002] could perhaps succeed in modeling this structure but this method is beyond the scope of this analysis. The wavelike pattern shown in Figure 10d, however, could be fitted by the force-free field model and is indicative of yet another flux rope passing by the spacecraft after the main ICME, the 

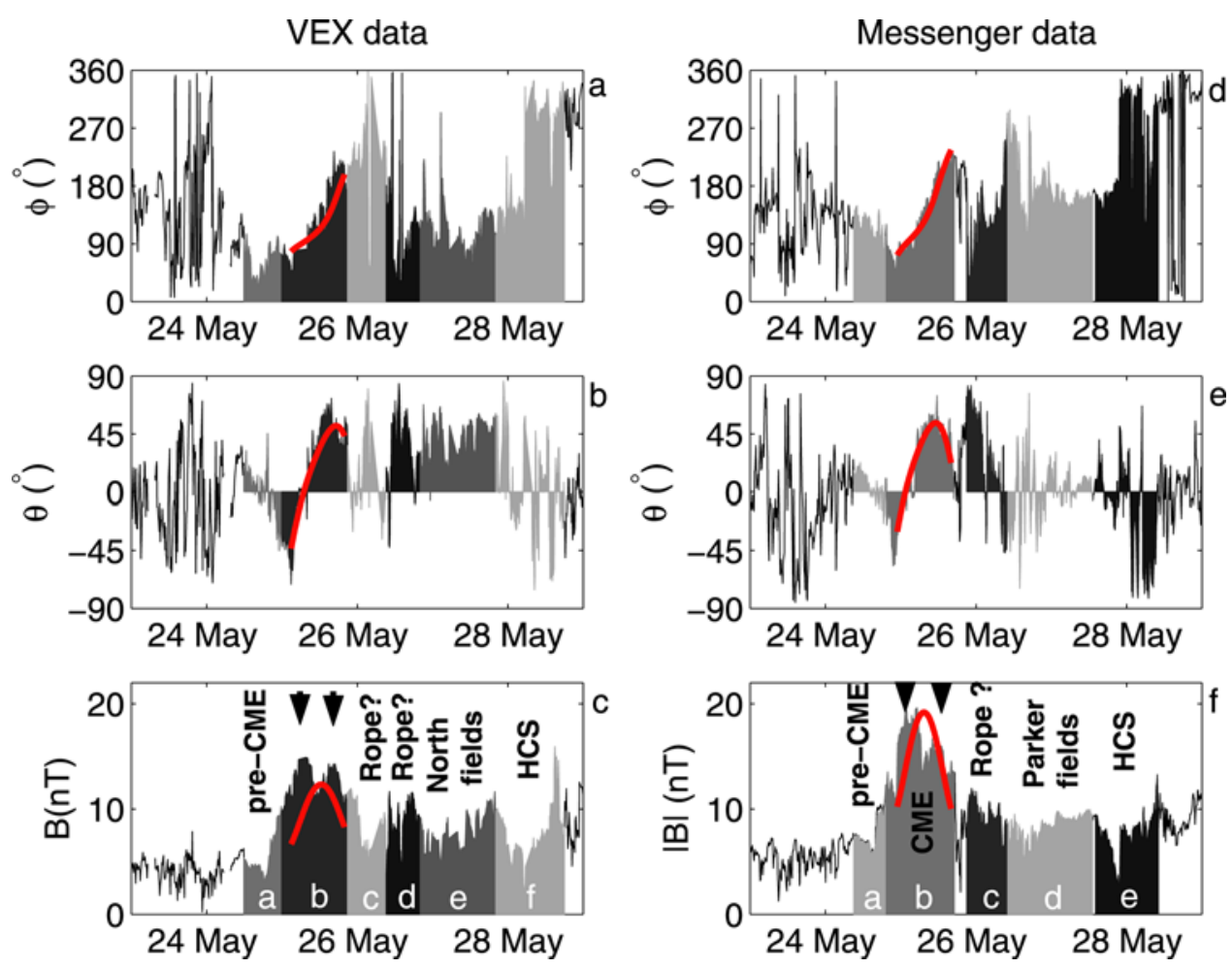

Figure 11. A comparison of the magnetic field azimuth, elevation angles, and strength measured by VEX and MESSENGER during the solar storm passage. The shaded areas mark the regions which show distinct magnetic field distributions in $(\phi, \theta)$ plots in each data set. Each region is plotted separately in Figures 10 and 12; the correspondence between the shaded areas of Figure 11 and the different plots of Figures 10 and 12 are indicated by the white letters at the base of each area in Figures 11c and 11f.

interval of data corresponding to this latter rotation of the magnetic field was, however, very short making this fit less significant than the main ICME event.

[44] We conclude from the analysis of the VEX magnetic field that highly ordered magnetic structures pass the spacecraft in the following chronological order: (1) deflected fields preceding the CME (Figure 10a), (2) a large-scale rotation of the magnetic field consistent with a flux rope bounded by the arrival of two dense plasma elements (Figure 10b), (3) two sets of rotations of the magnetic field, the second of which is consistent with a flux rope topology (Figures 10c and 10d), (4) a long-lasting period of north pointing fields (Figure 10e), and (5) a heliospheric current sheet crossing coincident with a CIR (Figure 10f). The main rotation was successfully associated with features $\mathrm{A}$ and $\mathrm{B}$. The lack of strong white light signals in the $\mathrm{HI}$ images from the other smaller events (identified by COR-2A) means that we could not track them accurately to Venus. Moreover, we have no estimate of the longitude of propagation of these smaller events. To interpret the observations made by VEX in terms of the continuous tracking of coronal outflows by COR-2A we had to make the necessary assumption that the smaller events were also propagating to Venus. We have mapped the structures seen by Venus Express ballistically back to COR-2A using the closest speed estimate from ASPERA-4 for each magnetic structure identified in Figure 10. The association is tentative; we find that the field variations seen in Figure 10c map ballistically to the emergence of structures $\mathrm{C}, \mathrm{D}$ and $\mathrm{E}, \mathrm{F}$ which emerge in very close proximity. The magnetic field in Figure 10d maps back ballistically to the structures $G$ and $H$. It is impossible in this highly complex sequence of events to associate any one of these small-scale in situ structures to their exact white light origin in COR-2A. We have established, however, that the complexity of the outflow at COR$2 \mathrm{~A}$ is followed by a highly structured and complex series of individual magnetic field rotations which are not inconsistent with flux rope topologies. We present, in the next part, the MESSENGER observations of the pre-CME, CME, and post-CME magnetic field variations. The MESSENGER spacecraft does not obtain full particle measurements and therefore the following description of the in situ observation is briefer.

\subsection{MESSENGER}

[45] The MErcury Surface, Space ENvironment, GEochemistry and Ranging (MESSENGER) probe is a NASA spacecraft, launched 3 August 2004 to study the characteristics and environment of Mercury from orbit. To make the mission feasible, MESSENGER makes extensive use of gravity assist maneuvers, thereby reducing the amount of rocket fuel needed to slow down. A second flyby of Venus was made at 2308 UT on 5 June 2007 at an altitude of 338 kilometers. At the time of ICME impact (25 May 2007), MESSENGER was therefore located very near Venus at a radial distance of 0.67AU (compare that with VEX: $0.72 \mathrm{AU}$ ) and $1.91^{\circ}$ of latitude north of VEX. The two spacecraft were $1.07^{\circ}$ apart in solar longitude. The magnetometer on the MESSENGER spacecraft is a low-noise, triaxial, fluxgate instrument with its sensor mounted on a $3.6 \mathrm{~m}$ long 

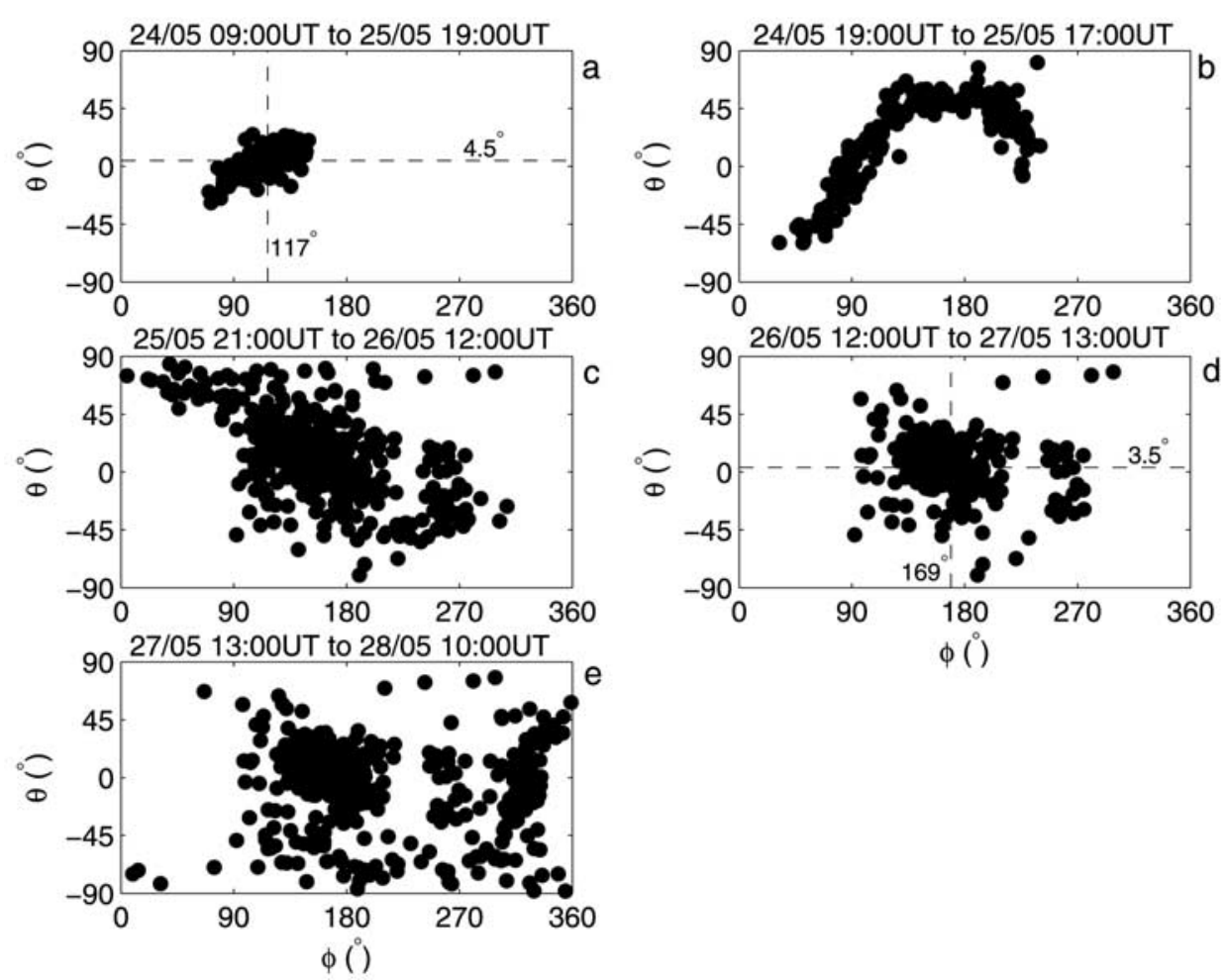

Figure 12. The analysis of the distribution of the magnetic field measured by MESSENGER in the same format as Figure 10.

boom [Anderson et al., 2007]. The Energetic Particle Spectrometer (EPS) and Fast Imaging Plasma Spectrometer (FIPS) sensors on the Energetic Particle and Plasma Spectrometer (EPPS) instrument [Andrews et al., 2007] are the plasma instruments onboard MESSENGER but unfortunately they cannot observe the solar wind because of the Sunward shield. In the following brief discussion we concentrate on the magnetic field observation. The MESSENGER observations of the azimuth, elevation and magnitude of the interplanetary magnetic field in RTN coordinates during the ICME event are shown in Figures $11 \mathrm{~d}, 11 \mathrm{e}$, and 11f, respectively. The field rotations observed by VEX and MESSENGER were nearly identical indicating that the two spacecraft passed through the same ICME region.

[46] Like VEX, MESSENGER measured a succession of large-scale rotations of the magnetic field as seen in elevation versus azimuth plots shown in Figure 12. Magnetic fields (Figure 12a) with mean azimuth, $\bar{\phi}=117^{\circ}$, and mean elevation, $\bar{\theta}=4.5^{\circ}$ (i.e., nearly located in the RT plane) are distributed at a significant angle off the nominal spiral field orientation (i.e., $\left(\phi=145^{\circ}, \theta=0^{\circ}\right),\left(\phi=325^{\circ}\right.$, $\left.\theta=0^{\circ}\right)$ ). This magnetic field is observed ahead of the flux rope which is nearly identical to the main rope observed at VEX with a full rotation of the magnetic field (Figure 12b). A period of large-scale fluctuations immediately follows exhibiting large excursions from the R-T plane (Figure 12c). Finally a current sheet crossing is also observed and is correlated in time with the expected arrival of the same CIR which hits VEX a few hours later (Figure 12e). Unlike VEX, MESSENGER does not measure a period of permanent north pointing magnetic fields during the corresponding interval (26 May 20071200 UT to 27 May 20071300 UT) (Figure 12d). Instead, MESSENGER observes in-ecliptic fields of average azimuthal angle $\bar{\phi}=169^{\circ}$ which are very close to the nominal Parker spiral orientation and, as such, we interpret them as undisturbed interplanetary magnetic fields.

[47] A force-free magnetic field with constant $\alpha$, was also used to describe the magnetic field in Figure 12b. A good fit of the field was obtained with an axis orientation in RTN coordinates given by $[-0.27,0.59,0.76]$. This rope is of the same chirality as the one observed at VEX and has a very similar rope axis orientation. The axis of the rope measured by MESSENGER has a larger component in the radial direction than the rope seen at VEX. The limitations of the VEX data imposed by the $3 \mathrm{~h}$ passage of VEX through the magnetosphere could have affected the quality of the fit on the VEX data and may help explain the differences.

[48] The type of tentative association carried out between the successive passages of magnetic field structures seen at Venus with the COR-2A images could not be done with the MESSENGER data because of the lack of available solar wind plasma moments and therefore preventing a prediction of the time of passage of each plasma element in COR-2. We note that the near absence of large-scale rotations of the magnetic field following the main event in the MESSENGER data was expected as posteruptive transients $(E, F, G, H)$ were propagating south of the SunMESSENGER line in COR-2A images and could have not impacted MESSENGER.

[49] A schematic comparison of rope axis orientation at the Sun (S), Venus Express (V) and MESSENGER (M) is presented in Figure 13. The orbit of Venus is plotted in 


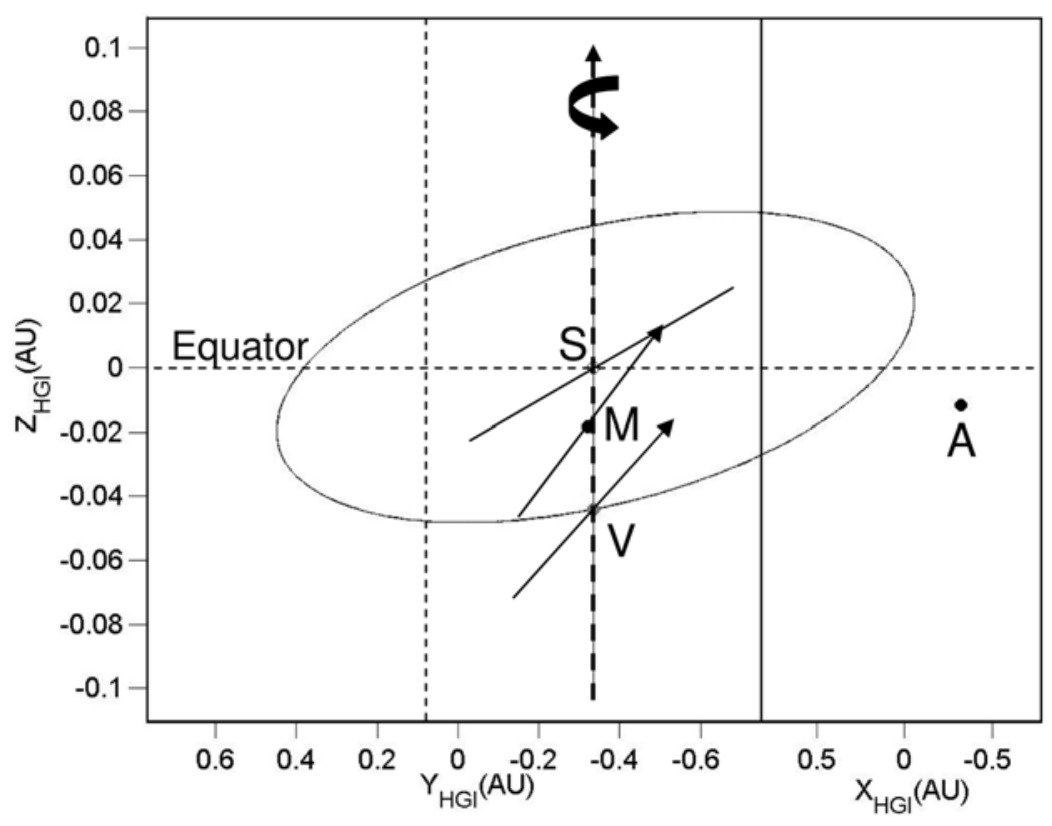

Figure 13. A view of the inner solar system along the longitude of Venus. The viewer is located in the equatorial plane (the horizontal dotted line passing through the center of the plot) and is looking toward the Sun. The proximity of MESSENGER (M) to Venus means that the Sun (S), M, and V are nearly aligned, whereas STEREO A, located $60^{\circ}$ west off this meridian, is located far to the right. The orbit of Venus is plotted in HGI coordinates. This plot format allows the comparison of the flux rope axis orientation obtained by white light analysis in Figure 3 with the in situ flux rope fittings in Figure 11.

Heliographic Inertial (HGI) coordinate system, the view of the inner solar system is from an observer located in the equatorial plane, looking toward the Sun along the Venus longitude. The inclined lines show the tilt of the main CME flux rope axis relative to the solar equator derived by white light images and by in situ force-free field fits. For the force-free field fit, the central axis orientation of the coaxial cylinders on which the helical field is distributed is marked by arrows. The in situ observations show that the flux rope axis had a significant horizontal component which was suggested by the modeling of white light images.

\section{Discussion}

[50] This paper sets out to interpret the complicated SECCHI observations of a solar storm, by using models of the solar corona and in situ spacecraft observations. COR-1/2A images revealed the complexity of the storm near onset, revealing that it consists of a variety of transient structures. These structures included CMEs, smaller loops, V-shaped density enhancements and blobs of plasma, all of which have been studied in white light images in detail during the last decade. Mysteries prevail, however, concerning the origin and nature of each of these structures. The present detailed study provides new evidence suggesting the following:

[51] 1. The V-shaped structure observed in white light at the rear of $\mathrm{CME}$ events can be explained by white light simulations of the corona as shown by Thernisien et al. [2006] and Wang and Sheeley [2006] and its passage is correlated in time with the end of the flux rope observed in situ.
[52] 2. The orientation of the simulated flux rope in SECCHI images suggests that the event is partly observed edge-on and this is consistent with the orientation of the magnetic flux rope observed in situ.

[53] 3. The density of plasma surrounding the rope can be used to track the evolution of the extent of ICME magnetic fields as they propagate out into the corona. In demonstrating these previous points, we have revealed how SECCHI can be used to study the time evolution of CME topology.

[54] According to this analysis, the presence and intensification of the leading loop-like structure (A) and the Vshaped structure (B) cannot be due to the transient plowing into the interplanetary medium because the event was slower than the ambient solar wind. The ASPERA-4 data shows that the speed of the ICME which passed Venus was decreasing during its passage. The speed of the transient at the onset of the flux rope (25 May 20070000 UT as estimated from the analysis of magnetic field organization in Figure 10) was $385 \mathrm{~km} \mathrm{~s}^{-1}, 30 \mathrm{~km} \mathrm{~s}^{-1}$ faster than the speed at the rear of the flux rope (25 May 20072100 UT). This is not unusual; Burlaga et al. [1981] and Klein and Burlaga [1982] showed many years ago that the speed typically decreases across magnetic clouds. By necessity a MC associated with such a speed profile will expand during its radial propagation. While the radial expansion of the CME (i.e., in latitude and longitude) works to alter the force-free field topology of the CME, the tension forces act to preserve the force-free field topology by forcing an expansion of the CME structure in the radial direction which pushes the surrounding plasma. In the present study we estimated an expansion speed in the radial direction of $\sim 30 \mathrm{~km} \mathrm{~s}^{-1}$ using $\mathrm{J}$ maps of HI images as well as in situ observations. The expansion of the minor radius of ICMEs 
was attributed by Suess [1988] to a kinematic effect whereby forces were acting to maintain part of the cylindrical aspect of CMEs. He noted that magnetic tension can be significant in magnetic clouds and that magnetic clouds do not therefore expand owing to magnetic overpressure, because the magnetic pressure imbalance can be counteracted by magnetic tension (pinch effect).

[55] In a more recent and comprehensive study, Lepping et al. [2008] investigated the expansion speed of a set of 53 well chosen Wind magnetic clouds that occurred over the period from early 1995 to April 2006. The most probable value of expansion speed from their two methods of estimation was shown to be around $\sim 35 \mathrm{~km} \mathrm{~s}^{-1}$ which is in good agreement with the expansion speeds derived from our techniques. We suggest that this expansion will force an increase in the speed of structure $A$ (at the front) and a decrease in the speed of structure B (at the rear). The consequential accumulation of plasma around the rope could be the reason why loop-like structures and large-scale V-shaped structures are so prominent in many events in HI. A similar theory was proposed by Vourlidas et al. [2000] to explain mass pileup at the rear of ICMEs in their analysis of the energetics of CMEs observed in LASCO coronagraph images. Colaninno and Vourlidas [2006] measured higher CME expansion speeds than the expansion calculated in this paper. The expansion speeds measured in this paper were done high in the corona which could suggest various regimes of expansions during the outward propagation of the flux rope. Events which are observed reliably from COR-1 all the way to HI-2 could be used to investigate this hypothesis. We note that additional effects such as an ICME plowing into the interplanetary medium [Kahler and Webb, 2007] or reconnection of the footpoints of CMEs [Wang and Sheeley, 2006] could act to alter the apparent morphology of CMEs as seen in white light images of other types of CME events.

[56] Sometimes ICMEs are composed of multiple CMEs such that interaction can occur [Burlaga et al., 1987; Wang et al., 2002]. Wang et al. [2002] presented a case of four ICMEs merging into a series of complex magnetic field events by the time they reached the ACE spacecraft. The brightness intensification of the $\mathrm{V}$-shaped structure during its outward propagation from COR-2A to HI could also be due to the posteruptive loops interacting with the rear of the main CME event, forcing a plasma pileup along the Sunward part of the main CME flux rope and eventually leading to the complex sequence of magnetic field rotations observed in situ. Such CME interactions were observed by Gopalswamy et al. [2001] in LASCO coronagraph images. Other mechanisms such as the formation of posteruptive current sheets can also lead to the release of V or Y-shaped structures [Lin and Forbes, 2000].

[57] The lack of in situ plasma data at VEX and MESSENGER has put constraints on how well the complicated environment surrounding the CME could be interpreted. The pre and post-CME flows at VEX and MESSENGER observed by COR-2A J maps in Figure 4 suggested that Venus Express and MESSENGER would not observe the same post-CME flows and this is confirmed in part by the absence in the MESSENGER data of some of the flux rope signatures observed in the VEX data.
[58] This study shows that the location of the flux ropes of CMEs can be estimated directly from white light images. As a consequence, for well-defined CME events, the kinematic evolution of the flux rope can be determined from white light images. The work of Bothmer and Schwenn [1998] and Rees and Forsyth [2003] shows that the orientation of $75 \%$ of magnetic clouds measured in situ typically follow the orientation of bipolar active regions during successive solar cycles (dictated by Hale's law). In the not too distant future we may therefore be in a position to predict the polarity, orientation and location of flux ropes of some ICMEs in white light images. Such an achievement would be of critical importance for space weather predictions.

[59] Acknowledgments. We thank Angelos Vourlidas and Len Burlaga for their comments on the original manuscript. This work was funded by STFC (UK). The STEREO/SECCHI data are produced by a consortium of RAL (UK), NRL (USA), LMSAL (USA), GSFC (USA), MPS (Germany), CSL (Belgium), IOTA (France), and IAS (France). The Venus Express and MESSENGER missions are ESA and NASA missions, respectively.

[60] Amitava Bhattacharjee thanks David Webb and another reviewer for their assistance in evaluating this paper.

\section{References}

Anderson, B. J., M. H. Acua, D. A. Lohr, J. Scheifele, A. Raval, H. Korth, and J. A. Slavin (2007), The magnetometer instrument on MESSENGER, Space Sci. Rev., 131, 417-450.

Andrews, G. B., et al. (2007), The energetic particle and plasma spectrometer instrument on the MESSENGER spacecraft, Space Sci. Rev., 131, $523-556$

Barabash, S., et al. (2007), The analyser of space plasmas and energetic atoms (ASPERA-4) for the Venus Express mission, Planet. Space Sci. $55,1772-1792$

Belcher, J. W., and L. Davis Jr. (1971), Large-amplitude Alfvén waves in the interplanetary medium: 2, J. Geophys. Res., 76, 3534-3565.

Borovsky, J. E., and M. H. Denton (2006), Differences between CMEdriven storms and CIR-driven storms, J. Geophys. Res., 111, A07S08, doi:10.1029/2005JA011447.

Bothmer, V., and R. Schwenn (1998), The structure and origin of magnetic clouds in the solar wind, Ann. Geophys., 16, 1-24.

Brueckner, G. E., et al. (1995), The large angle spectroscopic coronagraph (LASCO), Sol. Phys., 162, 357-402.

Burlaga, L. F. (1988), Magnetic clouds and force-free fields with constant alpha, J. Geophys. Res., 93, 7217-7224.

Burlaga, L. F. (1990), Coalescence of recurrent streams of different sizes and amplitudes, J. Geophys. Res., 95, 4247-4255.

Burlaga, L. F., E. Sittler, F. Mariani, and R. Schwenn (1981), Magnetic loop behind an interplanetary shock: Voyager, Helios, and IMP 8 observations, J. Geophys. Res., 86, 6673-6684.

Burlaga, L. F., L. W. Klein, N. R. Sheeley Jr., D. J. Michels, R. A. Howard, M. J. Koomen, R. Schwenn, and H. Rosenbauer (1982), A magnetic cloud and a coronal mass ejection, Geophys. Res. Lett., 9, 1317-1320.

Burlaga, L. F., K. W. Behannon, and L. W. Klein (1987), Compound streams, magnetic clouds, and major geomagnetic storms, J. Geophys. Res., 92, 5725-5734.

Burlaga, L. F., A. Hewish, and K. W. Behannon (1991), Structure and evolution of compound streams at not greater than $1 \mathrm{AU}, J$. Geophys. Res., 96, 21,213-21,225.

Burlaga, L. F., N. F. Ness, F. B. McDonald, J. D. Richardson, and C. Wang (2003), Voyager 1 and 2 observations of magnetic fields and associated cosmic-ray variations from 2000 through 2001: 60-87 AU, Astrophys. J., 582, 540-549.

Cane, H. V., I. G. Richardson, and G. Wibberenz (1997), Helios 1 and 2 observations of particle decreases, ejecta and magnetic clouds, J. Geophys. Res., 102, 7075-7086.

Coates, A. J., et al. (2008), Ionospheric photoelectrons at Venus: Initial observations by ASPERA-4 ELS, Planet. Space Sci., 56, 802-806.

Colaninno, R. C., and A. Vourlidas (2006), Analysis of the velocity field of CMEs using optical flow methods, Astrophys. J., 652, 1747-1754.

Cremades, H., and V. Bothmer (2004), On the three-dimensional configuration of coronal mass ejections, Astron. Astrophys., 422, 307-322. 
Crooker, N. U., and D. F. Webb (2006), Remote sensing of the solar site of interchange reconnection associated with the May 1997 magnetic cloud, J. Geophys. Res., 111, A08108, doi:10.1029/2006JA011649.

Crooker, N. U., G. L. Siscoe, S. Shodhan, D. F. Webb, J. T. Gosling, and E. J. Smith (1993), Multiple heliospheric current sheets and coronal streamer belt dynamics, J. Geophys. Res., 98, 9371-9381.

Davies, J. A., R. A. Harrison, A. P. Rouillard, N. R. Sheeley Jr., C. H. Perry, D. Bewsher, C. J. Davis, C. J. Eyles, S. R. Crothers, and D. S. Brown (2009), A synoptic view of solar transient evolution in the inner heliosphere using the Heliospheric Imagers on STEREO, Geophys. Res. Lett. 36, L02102, doi:10.1029/2008GL036182.

Eyles, C. J., et al. (2009), The Heliospheric Imager onboard the STEREO spacecraft, Sol. Phys., 254, 387-445.

Farrugia, M. W., M. W. Dunlop, F. Geurts, A. Balogh, D. J. Southwood, D. A. Bryant, and M. Neugebauer (1990), An interplanetary planar magnetic structure oriented at a large (about $80 \mathrm{deg}$ ) angle to the Parker spiral, Geophys. Res. Lett., 17, 1025-1028.

Gopalswamy, N., S. Yashiro, M. L. Kaiser, R. A. Howard, and J.-L. Bougeret (2001), Radio signatures of coronal mass ejection interaction: Coronal mass ejection cannibalism?, Astrophys. J., 548, L91-L94.

Howard, R. A., et al. (2008), Connection coronal and heliospheric investigation (SECCHI), Space Sci. Rev., 136, 67-115.

Hudson, H. S., J.-L. Bougeret, and J. Burkepile (2006), Coronal mass ejections: Overview of observations, Space Sci. Rev., 123, 13-30.

Kahler, S. W., and D. F. Webb (2007), V arc interplanetary coronal mass ejections observed with the Solar Mass Ejection Imager, J. Geophys. Res., 112, A09103, doi:10.1029/2007JA012358.

Klein, L. W., and L. F. Burlaga (1982), Interplanetary magnetic clouds at 1 AU, J. Geophys. Res., 87, 613-624.

Lavraud, B., and J. E. Borovsky (2008), Altered solar wind-magnetosphere interaction at low Mach numbers: Coronal mass ejections, J. Geophys. Res., 113, A00B08, doi:10.1029/2008JA013192.

Lavraud, B., M. F. Thomsen, J. E. Borovsky, M. H. Denton, and T. I Pulkkinen (2006), Magnetosphere preconditioning under northward IMF: Evidence from the study of coronal mass ejection and corotating interaction region geoeffectiveness, J. Geophys. Res., 111, A09208, doi:10.1029/2005JA011566.

Lepping, R. P., L. F. Burlaga, and J. A. Jones (1990), Magnetic field structure of interplanetary magnetic clouds at $1 \mathrm{AU}$, J. Geophys. Res., 95, 11,957-11,965.

Lepping, R. P., C.-C. Wu, D. B. Berdichevsky, and T. Fergusen (2008), Estimates of magnetic cloud expansion at 1 AU, Ann. Geophys., 26, 1919-1933.

Lin, J., and T. G. Forbes (2000), Effects of reconnection on the coronal mass ejection process, J. Geophys. Res., 105, 2375-2392.

Nakagawa, T., A. Nishida, and T. Saito (1989), Planar magnetic structures in the solar wind, J. Geophys. Res., 94, 11,761-11,775.

Neugebauer, M., D. R. Clay, and J. T. Gosling (1993), Planar magnetic structures in the solar wind, J. Geophys. Res., 98, 9383-9389.

Pulkkinen, T. I., N. Partamies, K. E. J. Huttunen, G. D. Reeves, and H. E. J. Koskinen (2007), Differences in geomagnetic storms driven by magnetic clouds and ICME sheath regions, Geophys. Res. Lett., 34, L02105, doi:10.1029/2006GL027775.

Rees, A., and R. J. Forsyth (2003), Magnetic clouds with east/west orientated axes observed by Ulysses during solar cycle 23, Geophys. Res. Lett., 30(19), 8030, doi:10.1029/2003GL017296.

Rees, A., and R. J. Forsyth (2004), Two examples of magnetic clouds with double rotations observed by the Ulysses spacecraft, Geophys. Res. Lett., 31, L06804, doi:10.1029/2003GL018330.

Rouillard, A. P., et al. (2008), First imaging of corotating interaction regions using the STEREO spacecraft, Geophys. Res. Lett., 35, L10110, doi:10.1029/2008GL033767.

Rouillard, A. P., et al. (2009), A multispacecraft analysis of a small-scale transient entrained by solar wind streams, Sol. Phys., 256, 307-326, doi:10.1007/s11207-009-9329-6.
Schwenn, R., et al. (2006), Coronal observations of CMEs: Report of working group A, Space Sci. Rev., 123, 127-176.

Sheeley, N. R., Jr., R. A. Howard, D. J. Michels, M. J. Koomen, R. Schwenn, K. H. Muehlhaeuser, and H. Rosenbauer (1985), Coronal mass ejections and interplanetary shocks, J. Geophys. Res., 90, 163-175.

Sheeley, N. R., Jr., et al. (1997), Measurements of flow speeds in the corona

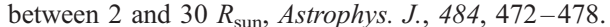

Sheeley, N. R., J. H. Walters, Y. M. Wang, and R. A. Howard (1999), Continuous tracking of coronal outflows: Two kinds of coronal mass ejections, J. Geophys. Res., 104, 24,739-24,768.

Sheeley, N. R., Jr., et al. (2008a), SECCHI observations of the Sun's garden-hose density spiral, Astrophys. J., 674, L109-L112.

Sheeley, N. R., Jr., et al. (2008b), Heliospheric images of the solar wind at Earth, Astrophys. J., 675, 853-862.

St. Cyr, O. C., et al. (2000), Properties of coronal mass ejections: SOHO LASCO observations from January 1996 to June 1998, J. Geophys. Res., $105,18,169-18,186$.

Suess, S. T. (1988), Magnetic clouds and the pinch effect, J. Geophys. Res., 93, 5437-5445.

Suess, S. T., A. Bemporad, and G. Poletto (2004), A slow streamer blowout at the Sun and Ulysses, Geophys. Res. Lett., 31, L05801, doi:10.1029/ 2003GL018895.

Thernisien, A. F. R., R. A. Howard, and A. Vourlidas (2006), Modeling of flux rope coronal mass ejections, Astrophys. J., 652, 763-773.

Vourlidas, A., and R. A. Howard (2006), The proper treatment of coronal mass ejection brightness: A new methodology and implications for observations, Astrophys. J., 642, 1216-1221.

Vourlidas, A., P. Subramanian, K. P. Dere, and R. A. Howard (2000), Large-angle spectrometric coronagraph measurements of the energetics of coronal mass ejections, Astrophys. J., 534, 456-467.

Wang, Y.-M., and N. R. Sheeley Jr. (2006), Observations of flux rope formation in the outer corona, Astrophys. J., 650, 1172-1183.

Wang, Y.-M., S. Wang, and P. Z. Ye (2002), Multiple magnetic clouds in interplanetary space, Sol. Phys., 211, 333-344.

Webb, D., and R. A. Howard (1994), The solar cycle variation of coronal mass ejections and the solar wind mass flux, Space Sci. Rev, 99, 4201-4220

Zhang, T. L., et al. (2006), Magnetic field investigation of the Venus plasma environment: Expected new results from Venus Express, Planet. Space Sci., 54, 1336-1343.

B. Anderson, Johns Hopkins University Applied Physics Laboratory, Baltimore, MD 20723, USA

D. Bewsher, S. R. Crothers, C. J. Davis, J. A. Davies, C. J. Eyles, M. Hapgood, and R. A. Harrison, Space Science and Technology Department, Rutherford Appleton Laboratory, Chilton OX11 0QX, UK.

D. S. Brown, M. Grande, and I. Whittaker, Institute of Mathematics and Physics, Aberystwyth University, Aberystwyth SY23 3BZ, UK.

C. M. Carr, R. J. Forsyth, and N. P. Savani, Space and Atmospheric Physics, Blackett Laboratory, Imperial College London, London SW7 2BW, UK.

A. J. Coates, G. H. Jones, and S. Tsang, Mullard Space Science Laboratory, University College London, Dorking RH5 6NT, UK.

R. A. Frahm and J. D. Winningham, Southwest Research Institute, San Antonio, TX 78238, USA.

M. Fränz, Max-Planck-Insitut für Sonnensystemforschung, KatlenburgLindau D-37191, Germany.

R. A. Howard, N. R. Sheeley, and A. Thernisien, Space Science Division, Naval Research Laboratory, Washington, DC 20375-5352, USA.

M. Lockwood and A. P. Rouillard, Space Environment Physics Group, School of Physics and Astronomy, University of Southampton, Southampton SO17 1BJ, UK. (alexisrouillard@yahoo.co.uk)

T.-L. Zhang, Space Research Institute, Austrian Academy of Sciences, Graz A-8042, Austria. 